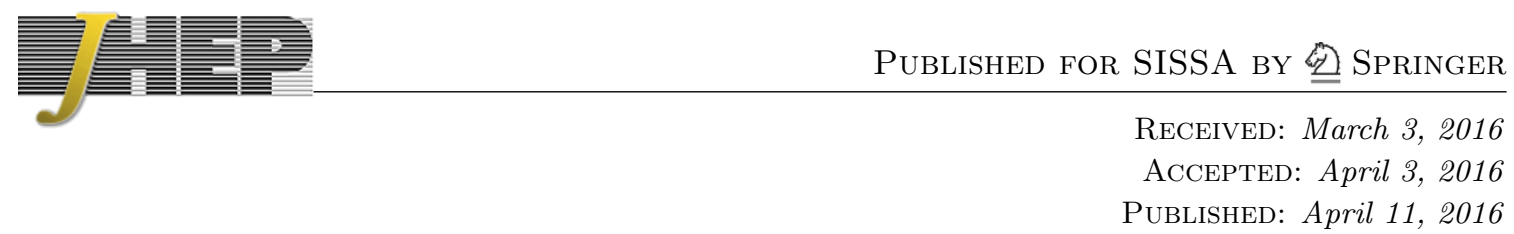

\title{
Long-lived staus and displaced leptons at the LHC
}

\author{
Jared A. Evans and Jessie Shelton \\ Department of Physics, University of Illinois at Urbana-Champaign, \\ Urbana, IL 61801, U.S.A. \\ E-mail: jaredaevans@gmail.com, sheltonj@illinois.edu
}

ABSTRACT: As the majority of LHC searches are focused on prompt signatures, specific long-lived particles have the potential to be overlooked by the otherwise systematic new physics programs at ATLAS and CMS. While in many cases long-lived superparticles are now stringently constrained by existing exotic searches, we point out that the highly motivated model of gauge mediation with staus as the next-to-lightest superparticle (NLSP) is relatively far less tested. We recast LHC searches for heavy stable charged particles, disappearing tracks, and opposite-flavor leptons with large impact parameters to assess current constraints on a variety of spectra that contain an NLSP stau, and find that portions of the parameter space motivated by naturalness are still experimentally unexplored. We additionally note a gap in the current experimental search program: same-flavor leptons with large impact parameters evade the suite of existing searches for long-lived objects. This gap is especially noteworthy as vetoes on displaced leptons in prompt new physics searches could be systematically discarding such events. We discuss several motivated models that can exhibit same-flavor displaced leptons: gauge mediation with co-NLSP sleptons, extended gauge mediation, R-parity violation, and lepton-flavored dark matter that freezes in during a matter-dominated era of the early universe. To address this gap, we propose a straightforward extension of the CMS search for leptons with large impact parameters, and project sensitivity to these scenarios at $13 \mathrm{TeV}$. Throughout this analysis, we highlight several methods whereby LHC searches for exotic long-lived objects could potentially improve their sensitivity to the displaced leptons originating from gauge mediation and beyond.

KeYwords: Supersymmetry Phenomenology

ARXIV EPRINT: 1601.01326 


\section{Contents}

1 Introduction 1

2 LHC sensitivity to long-lived staus $\quad 3$

2.1 CMS Heavy Stable Charged Particle Search 4

2.2 Disappearing track searches 5

$\begin{array}{lll}2.2 .1 & \text { ATLAS disappearing tracks } & 6\end{array}$

$\begin{array}{lll}2.2 .2 & \text { CMS disappearing tracks } & 8\end{array}$

2.3 CMS Displaced $e \mu$ search 9

$\begin{array}{ll}2.4 \text { Constraints on long-lived staus } & 10\end{array}$

3 Models with displaced same-flavor leptons $\quad \mathbf{1 5}$

$\begin{array}{lll}3.1 & \text { Slepton Co-NLSP } & 16\end{array}$

$\begin{array}{lll}3.2 & \text { Sleptons in extended gauge mediation } & 16\end{array}$

$\begin{array}{lll}3.3 & R \text {-parity violating decays of staus via LLE operators } & 17\end{array}$

3.4 Lepton-flavored dark matter from freezein 18

$\begin{array}{lll}4 & \text { A search for displaced same-flavor leptons } & 19\end{array}$

$\begin{array}{lll}5 & \text { Discussion and conclusions } & 23\end{array}$

$\begin{array}{ll}\text { A Validation of recasting procedures } & 25\end{array}$

B Details of the Extended Gauge Mediation Model 26

C Freezein during an early matter-dominated era 29

\section{Introduction}

Run I at the LHC has been a phenomenal success. However, despite pressure from naturalness for new electroweak scale particles, no such particles have been observed yet by the LHC experiments (see e.g., [1]). Weak-scale supersymmetry (SUSY), the longstanding front-runner to explain the stability of the electroweak scale, has been subjected to more and more stringent constraints as time progresses. The parameter spaces for natural gluinos, stops, and even electroweakinos are now highly constrained across a wide variety of spectra. The current absence of clear signals of new physics confronts us with three logical possibilities concerning weak-scale superpartners: (i) they are not there to be found; (ii) they are just around the corner (and will hopefully be seen at Run II); or (iii) they are hidden somehow from the existing array of searches. A variety of mechanisms have been devised over the years in order to hide supersymmetry from collider searches, 
but natural spectra in the majority of these scenarios are now under pressure from the comprehensive search programs at ATLAS and CMS [2]. Even mechanisms such as $R$-parity violation [3] or stealth [4] that can hide SUSY from traditional $\not_{T}$-based searches are already significantly at odds with the signals expected from natural Majorana gluinos [5], although counter examples exist $[5,6]$.

New particles with macroscopic decay lengths, as can easily arise in gauge-mediated SUSY breaking (GMSB) [7], $R$-parity violating (RPV) SUSY, mini-split SUSY [8], and other models (e.g., [9]), can potentially elude the selection criteria for the standard suite of collider searches, but, thanks to dedicated searches for long-lived objects, are now often more constrained than their prompt counterparts [10-12]. However, models predicting solitary displaced leptons are less thoroughly constrained. Solitary displaced leptons are leptons that originate from a displaced vertex that has no other visible decay products, i.e., from a long-lived particle that decays to an invisible particle and a lepton that does not point back to the primary vertex. While displaced leptons figure in many existing searches, almost all of these searches look for a vertex containing a displaced lepton together with at least one other object, such as another lepton [13-15] or $\geq 4$ additional tracks $[15,16]$; the only existing search sensitive to solitary displaced leptons is the CMS search for a displaced opposite-sign $e-\mu$ pair [17]. Models predicting solitary displaced leptons can be surprisingly invisible to current searches, as lepton quality requirements in most prompt searches veto leptons with impact parameters down to just a few hundred microns, and often discard entire events with cosmic-ray-motivated vetoes on muons with large impact parameters.

Solitary displaced leptons arise in many theories. Perhaps the best-motivated examples arise from theories of GMSB, which frequently predict spectra where the right-handed stau is the next-to-lightest superpartner (NLSP). Although minimal models of GMSB have difficulty accommodating the heavy Higgs mass of $125 \mathrm{GeV}[18,19]$ without introducing large tuning [20], extensions to GMSB can readily account for this while approaching the minimal fine tuning possible in the MSSM, see e.g., [21-28]. Adding new fields to the MSSM can also raise $m_{h}$, either by GMSB-specific mechanisms, e.g., [29-32], or with modular modifications to the SUSY Higgs sector, such as non-decoupling $D$-terms [33-38]. The lifetime of a slepton NLSP decaying via $\tilde{\ell} \rightarrow \ell \tilde{G}$ can be expressed as

$$
c \tau \approx 100 \mu \mathrm{m}\left(\frac{100 \mathrm{GeV}}{m_{\tilde{\tau}}}\right)^{5}\left(\frac{\sqrt{F}}{100 \mathrm{TeV}}\right)^{4} .
$$

Even for a relatively low SUSY-breaking scale $\sqrt{F} \sim 100 \mathrm{TeV}$, the decay lengths of such sleptons border on being displaced at the LHC. For high SUSY-breaking scales, these sleptons become detector-stable and fall under the purview of searches for heavy stable charged particles (HSCPs). In the intermediate regime, which spans roughly four orders of magnitude in lifetime ( $c \tau \sim 100 \mu \mathrm{m}-1 \mathrm{~m}$ ), where the slepton lives too briefly to survive the detector, but long enough to be vetoed in many standard prompt SUSY searches, the resulting signature is opposite-sign taus originating a macroscopic distance away from the primary interaction point. If the lifetime of the slepton is sufficiently long (at the LHC, $c \tau \sim$ $\mathcal{O}(50 \mathrm{~cm}))$, it is possible to search directly for the track left by the slepton. In the eyes of the 
tracking algorithm, the slepton will disappear if it decays before reaching the calorimeter, making the primary signal of a stau in this lifetime range a kinked or disappearing track. For shorter slepton lifetimes $(100 \mu \mathrm{m} \lesssim c \tau \lesssim 5 \mathrm{~cm})$, it is the displaced daughter leptons that drive search sensitivity. At LEP2, dedicated long-lived slepton searches covered this entire range of signatures, where OPAL [39] sets the best limits. At the LHC, HSCP searches [4042], searches for disappearing tracks [43, 44], and the CMS search for displaced $e^{ \pm} \mu^{\mp}[17]$ (henceforth, "CMS displaced $e \mu$ ") together target the range of displaced slepton signatures, but in general do not specifically target sleptons, and are not optimized for them.

Moreover, several classes of theories can give rise exclusively to displaced same-flavor leptons. In the context of SUSY, this signature can arise with RPV couplings or in extended gauge mediation. Outside of SUSY, lepton-flavored dark matter can provide an elegant mechanism to produce such signatures. All of these models can yield displaced signatures that are currently not covered by the existing array of LHC searches, highlighting an outstanding gap in the search coverage for new physics. Additionally, as we will demonstrate, a same-flavor displaced lepton search would significantly improve sensitivity to long-lived staus as well as theories with long-lived slepton co-NLSPs [45-47].

The aim of this paper is twofold. In section 2, we will establish existing collider constraints on long-lived staus by recasting the CMS displaced $e \mu$ search, the disappearing track searches at ATLAS and CMS, and a heavy stable charged particle search, thus obtaining a clear picture of current sensitivity to displaced decays of a stau NLSP. In addition to direct stau production, we also consider production in decay chains originating from gluinos, stops, and Higgsinos. In the course of this endeavor, we will discuss several possible modifications to the existing searches that could enhance sensitivity to displaced staus. Next, we discuss several concrete models in section 3 that give rise to same-flavor displaced lepton signatures. We propose a simple extension to the existing CMS displaced $e \mu$ search strategy to close the gap in LHC searches for displaced same-flavor leptons, and estimate the resulting sensitivity at $13 \mathrm{TeV}$ for several models in section 4. Extensions and modifications of existing search strategies that could potentially enhance sensitivity to displaced stau decays in particular and solitary displaced lepton signatures in general are summarized in the conclusions.

\section{LHC sensitivity to long-lived staus}

One of the most common predictions in models of GMSB is that the $\tilde{\tau}_{R}$ is the NLSP (with the gravitino as the LSP). Even at relatively low SUSY-breaking scales, the tiny width for $\tilde{\tau}_{R} \rightarrow \tau \tilde{G}$ can result in displaced leptons, as shown in (1.1). The best LEP2 limits on direct NLSP stau pair production come from OPAL, and exclude $\tilde{\tau}(\tilde{\mu})$ NLSPs below $87 \mathrm{GeV}(94 \mathrm{GeV})$ and become more stringent for longer lifetimes (up to $97 \mathrm{GeV}$ for both particles) [39]. Depending on the stau lifetime, the resulting collider signatures may yield:

- Opposite-sign solitary displaced leptons. A lepton's displacement is characterized by its impact parameter, which is typically defined as the minimum three-dimensional distance from the lepton track to the primary vertex, although in the CMS displaced 
$e \mu$ search [17] a two-dimensional impact parameter is used with respect to the center of the beampipe. The CMS displaced $e \mu$ search is the only existing LHC analysis with sensitivity to solitary displaced leptons.

- Disappearing tracks. At longer lifetimes $c \tau \sim \mathcal{O}(50 \mathrm{~cm})$, the $\tilde{\tau}$ will have left a reconstructable, short, high- $p_{T}$ track in the tracker. This places the stau in the territory of the disappearing track searches $[43,44]$. The signature in this range is really a kinked track, as was directly searched for at LEP [39]. In the busier environment offered by the LHC, however, the track associated with the daughter lepton is typically not reconstructed or may not be associated with the parent slepton track, and triggering on a kinked track is nearly impossible. The LHC disappearing track searches are only sensitive to the sleptons' visible decay products if they leave significant calorimeter deposits or make tracks in the muon chamber.

- HSCPs. Even longer lifetimes yield detector-stable charged particles, which have been directly searched for in refs. [40,41]. While we expect the ATLAS and CMS HSCP searches to have similar sensitivity, we choose to recast the CMS search, as detailed efficiency maps to facilitate recasting are provided [48]. ${ }^{1}$

The main objective of this section is to establish the current LHC coverage of long-lived staus from the various search strategies discussed above. In addition to direct stau production, we will consider staus produced in cascade decays originating from gluinos, stops, or Higgsinos. All of the benchmarks we consider are generated in Madgraph 5 [51] (using the TauDecay package [52]) and showered in Pythia 8 [53]. Hadrons are clustered according to the jet algorithms of the individual searches, and a simple jet energy smearing with resolution of $\sigma_{E}=0.05 \sqrt{E(\mathrm{GeV})}[54]$ is applied. The signal production cross-sections are fixed to the nominal NLO+NLL value as provided by ref. [55] or as computed in Prospino2 [56] or Resummino [57]. In the following subsections, we will describe the relevant searches in some detail with emphasis on our recasting procedure (for details of validation, see appendix A). The resulting constraints on spectra with a long-lived $\tilde{\tau}_{R}$ NLSP are collected in section 2.4.

\subsection{CMS Heavy Stable Charged Particle Search}

The CMS HSCP search [40] looks at a variety of models containing heavy charged particles that survive the detector. Of the various sub-analyses employed in this search, the most pertinent for long-lived staus is the "tracker + time-of-flight" sub-analysis, which requires a track to be reconstructed in both the inner tracker and the muon system. ${ }^{2}$ In this signal region, events are required to have at least one high-quality track with $|\eta|<2.1$

\footnotetext{
${ }^{1}$ Searches targeting particles that decay in the calorimeters or the muon system either explicitly veto events where a charged track in the inner detector points to the displaced decay or require multiple charged tracks at a displaced vertex, and thus are not sensitive to this class of signals $[49,50]$.

${ }^{2}$ Although the "tracker only" sub-analysis is motivated by charge-flipping scenarios, it could potentially provide greater sensitivity for shorter stau lifetimes. However, as the relevant lifetime window is expected to overlap with the range covered by disappearing track searches, and efficiency maps for this search region are not provided, we do not consider this possibility in detail here.
} 
and $p_{T}>70 \mathrm{GeV}$. This track must pass mild isolation criteria, with the sum of all nearby tracks $\sum p_{T, \Delta R<0.3}^{\text {trks }}<50 \mathrm{GeV}$ and $I_{\Delta R<0.3}^{\text {calo,track }}<0.3$, where

$$
I_{\Delta R<R}^{C, X}<Y
$$

means that in a $\Delta R=R$ cone around object $X$, the sum of either calorimeter deposits ( $C=$ calo) or charged tracks $(C=$ trks $)$ divided by the $p_{T}$ of $X$ must be less than $Y$. Additionally, this track needs to exceed some average $d E / d x$ value (see ref. [40] for details) and have a sufficiently low velocity $\beta$ satisfying $1 / \beta>1.225$.

While long-lived staus are one of the signal models considered in the CMS HSCP search [40], in order to understand the sensitivity of this search for staus with general values of $\left(m_{\tilde{\tau}}, c \tau\right)$, and to establish results for staus appearing at the end of cascade decays, we need to recast the search. To do so, we follow the detailed instructions provided in ref. [48] using only the $8 \mathrm{TeV}$ data. These instructions employ efficiency maps [58] that provide both an on- and off-line probability $\left(P^{\text {on }}, P^{\text {off }}\right)$ for an individual track to satisfy the basic requirements of the search as a function of $p_{T}, \eta, \beta$, and $m_{\tilde{\tau}}$. We then scale $P^{\text {on }}$ by the probability that a track survived through the muon chamber, $e^{-\frac{m_{\tilde{\tau}} x(\eta)}{p c \tau}}$, where $x(\eta)$ is a simple geometric approximation for the size of the detector: $x(|\eta| \leq 0.8)=900 \mathrm{~cm}$, $x(0.8<|\eta| \leq 1.1)=1000 \mathrm{~cm}, x(1.1<|\eta| \leq 2.1)=1100 \mathrm{~cm}$. As the efficiency maps do not include the effect of the isolation cuts, we set $P^{\text {off }}=0$ if either $\sum p_{T, \Delta R<0.3}^{\text {trks }}>50 \mathrm{GeV}$ or $I_{\Delta R<0.3}^{\text {calo,track }}>0.3$. The on- and off-line probabilities are combined to yield a total efficiency of

$$
P^{\text {net }}=\left(P_{1}^{\mathrm{on}}+P_{2}^{\mathrm{on}}-P_{1}^{\mathrm{on}} \times P_{2}^{\mathrm{on}}\right)\left(P_{1}^{\mathrm{off}}+P_{2}^{\mathrm{off}}-P_{1}^{\mathrm{off}} \times P_{2}^{\mathrm{off}}\right),
$$

where the two different probabilities correspond to the two different $\tilde{\tau}_{\mathrm{s}}$ in the event (with one track, it is simply $P^{\text {net }}=P^{\text {on }} P^{\text {off }}$ ). The signal falls into one of four signal regions based on $m_{\tilde{\tau}}$, which leads to a maximum number of allowed signal events used to set our 95\% exclusion contours:

$$
N_{95}= \begin{cases}21.6 & m_{\tilde{\tau}} \leq 166 \mathrm{GeV} \\ 8.3 & 166 \mathrm{GeV}<m_{\tilde{\tau}} \leq 330 \mathrm{GeV} \\ 3.0 & 330 \mathrm{GeV}<m_{\tilde{\tau}} \leq 500 \mathrm{GeV} \\ 3.0 & 500 \mathrm{GeV}<m_{\tilde{\tau}}\end{cases}
$$

Our modeling reliably reproduces the constraints from the search, so we assign the recommended $25 \%$ uncertainty to our modeling of this search in figures $1 \& 2$. Further details of the validation of our modeling are given in appendix A.

\subsection{Disappearing track searches}

Both ATLAS and CMS have searches for disappearing tracks, i.e., tracks of high quality within the inner layers of the tracker that suddenly vanish, leaving no hits in the outer layers of the tracker. Disappearing track signals are characteristic of long-lived nearly degenerate winos, as can arise in anomaly-mediated SUSY breaking (AMSB) [59,60], and the LHC searches are optimized for this model. These disappearing track searches can also 
be sensitive to long-lived $\tilde{\tau}_{R} \mathrm{~s}$, as the tracking algorithms are not directly sensitive to the visible decay products of charged particles that decay in flight.

For readability in our figures, we will present only the strongest limit from the two disappearing track searches. A breakdown of the individual sensitivities is presented in appendix A. Below we discuss the ATLAS [43] and CMS [44] searches in turn.

\subsubsection{ATLAS disappearing tracks}

The ATLAS disappearing track search [43] requires at least one hard jet with $p_{T}>90 \mathrm{GeV}$, large missing energy $\mathbb{E}_{T}>90 \mathrm{GeV}$, and a minimal azimuthal separation between the $E_{T}$ and the hardest two jets of $\Delta \phi^{\text {jet }-\not_{T}}>1.5$. The search additionally requires that there are no electron or muon candidates (satisfying loose ID requirements) in the event. Backgrounds containing muons are further suppressed by requiring no tracks in the muon calorimeter with $p_{T}>10 \mathrm{GeV}$.

After this basic selection, the search requires a high $p_{T}(>75 \mathrm{GeV})$ track stub of good quality that leaves hits in the inner tracker (pixel and silicon microstrip layers), but fewer than five hits in the straw-tube transition radiation tracker (TRT) occupying the outer tracker region with an inner (outer) radius of $56.3 \mathrm{~cm}(106.6) \mathrm{cm}^{3}{ }^{3}$ This disappearing track must be the highest $p_{T}$ track in the event, sit within the range $0.1<|\eta|<1.9$, be isolated from other tracks, $I_{\Delta R<0.4}^{\text {trks,track }}<0.04$, and separated from all jets with $p_{T}>45 \mathrm{GeV}$ by $\Delta R>0.4$.

In order to model the efficiency for a charged particle decaying within the tracker to leave a track that passes the selection requirements, we partition the tracker into $10-\mathrm{cm}$ bins of radial displacement, $L_{x y}$. Each bin is weighted with the probability $\mathcal{P}$ for the longlived particle to decay within that bin. As the disappearing track is required to have at least two hits in the silicon microstrip layers that begin near $L_{x y}=30 \mathrm{~cm}$, particles that decay before this have zero identification efficiency, $\epsilon_{I D}=0.0$. Starting at $30 \mathrm{~cm}$ of radial displacement we have $\epsilon_{I D}=1.0$ until the TRT starts at $56.3 \mathrm{~cm}$. After this, we model the number of TRT hits using a Poisson distribution based on how far the particle has propagated radially through the TRT. We assign an average of 25 hits to particles which survive the entire TRT; however, we set an efficiency floor of $\epsilon_{I D, \text { min }}=0.1$, following figure 2 of [43]. This floor allows for the increased sensitivity to the larger values of $c \tau$ that ATLAS observes. Our resulting simple modeling of the track identification efficiency is shown in table 1. Variations on the implementation of the track identification efficiency produced only small modifications to the ultimate sensitivity. The net probability that an event containing two long-lived staus will possess a disappearing track that passes the selection is

$$
w=\sum_{x \in \text { bins }} \epsilon_{I D}(x) \mathcal{P}_{1}(x) \mathcal{P}_{2}(<6 \mathrm{~m})+\sum_{x \in \text { bins }} \epsilon_{I D}(x) \mathcal{P}_{2}(x) \mathcal{P}_{1}(<30 \mathrm{~cm}),
$$

where $\mathcal{P}_{1}\left(\mathcal{P}_{2}\right)$ refers to the higher (lower) $p_{T}$ stau. The second term is the region where the lower- $p_{T}$ stau yields the hardest track in the event.

\footnotetext{
${ }^{3}$ For comparison, a typical charged particle leaves an average of 32 hits in the TRT [43].
} 


\begin{tabular}{|c|c|c|c|c|c|c|c|c|}
\hline$L_{x y}(\mathrm{~cm})$ & $<30$ & $30-50$ & $50-60$ & $60-70$ & $70-80$ & $80-90$ & $90-600$ & $>600$ \\
\hline ID Efficiency $\epsilon_{I D}$ & 0.0 & 1.0 & 0.99 & 0.91 & 0.52 & 0.18 & 0.1 & 0.0 \\
\hline
\end{tabular}

Table 1. Our simplified modeling of identification efficiencies in the ATLAS disappearing track search as a function of the radial displacement $L_{x y}$. We note that our limits are largely insensitive to the precise details of the modeling within the TRT.

This search has four non-exclusive signal regions, defined by the $p_{T}$ of the disappearing track, $p_{T}>75,100,150$, and $200 \mathrm{GeV}$, which have a maximum allowed number of signal events at $95 \%$ CL of $N_{95}=35.7,20.8,12.6$, and 8.9 observed $\left(N_{95, \exp }=28.8,21.3,13.6\right.$, and 11.3 expected), respectively. At each point we use the observed sensitivity from the bin with the best expected sensitivity to place constraints. This search has been validated on the AMSB wino model and has fairly good agreement, as shown in appendix A. Our recast yields weakened limits at high values of $c \tau$ relative to the experimental result; however, this limitation of our modeling will not be important for our conclusions.

Of course, the benchmark models we are considering have an additional layer of complication, namely that our $\tilde{\tau}_{R^{\mathrm{S}}}$ do not simply disappear, but yield an energetic decay product - an electron, muon, or hadronic tau - that can deposit energy in the calorimeter, appear as a jet, modify the $E_{T}$ distribution, and/or leave high $p_{T}$ tracks in the muon calorimeter. In order to simulate this, we model the $\tilde{\tau}$ decays as occurring at the center of the $10-\mathrm{cm}$ discrete bin of radial displacement. If the $\tilde{\tau}$ gives an electron or a hadronic tau with neutral pions ${ }^{4}$ (hadronic tau without neutral pions), we deposit the energy of the decay products at the point where the track emanating from the center of this bin intersects a cylinder going roughly halfway through the ECAL (HCAL) - we use $\{R, Z\}=\{175 \mathrm{~cm}, 420 \mathrm{~cm}\}(\{325 \mathrm{~cm}, 520 \mathrm{~cm}\})$ for ATLAS. This calorimeter deposit is labeled as a jet (photons are not distinguished from jets in this search), and the $90 \mathrm{GeV}$ jet, recalculated $E_{T}$, and various isolation requirements are checked with these reprocessed kinematics. If the stau decays within the calorimeter, we simply label it as a jet centered at the point where the track connected with the calorimeter. If a track survives far enough into the muon chamber (we use a six-meter radius), the event is assumed to be vetoed by the strict muon veto criteria of the ATLAS search.

Despite the successful validation of our recasting procedure for the original wino signal model, we stress that the additional complications due to the stau decay products greatly decrease the reliability of our modeling. Because of this, we present a $50 \%$ modeling uncertainty for this search. Nonetheless, our modeling is sufficiently accurate to demonstrate that disappearing track searches also have good sensitivity to "kinked track" signals. Implementing a full GEANT-based detector simulation in order to accurately treat this class of signals is important, but is best done by experimentalists.

\footnotetext{
${ }^{4}$ Hadronic taus with neutral pions also contain at least one charged pion, which typically deposits most of its energy into the HCAL. For simplicity, in this class of hadronic tau, we deposit all energy at the center of the ECAL for the purposes of determining the resulting position of the jet.
} 


\subsubsection{CMS disappearing tracks}

CMS also has a search looking for disappearing tracks, motivated by nearly-degenerate winos in AMSB [44]. In this search, CMS requires large missing energy of $\not_{T}>100 \mathrm{GeV}$, and at least one hard jet with $p_{T}>110 \mathrm{GeV},|\eta|<2.4$, which has at least $20 \%$ of its energy in charged hadrons, less than $70 \%$ in neutral hadrons or photons, and less than $50 \%$ in electrons. The hardest two jets and the $E_{T}$ must be azimuthally separated by $\Delta \phi^{\text {jet}-E_{T}}>0.5$, and all jets with $p_{T}>30$ and $|\eta|<4.5$ must be separated from one another by $\Delta \phi^{j j}<2.5$ to reduce QCD background.

The candidate disappearing tracks are required to be of high quality, have $p_{T}>50 \mathrm{GeV}$, and fall within an $\eta$ range of either $|\eta|<0.15,0.35<|\eta|<1.42$, or $1.85<|\eta|<2.1$. The tracks are required to be isolated, with no jets of $p_{T}>30 \mathrm{GeV}$ within $\Delta R$ of 0.5 , $I_{\Delta R<0.3}^{\text {trks, track }}<0.05$, and $E_{\text {calo }}^{\Delta R<0.5}<10 \mathrm{GeV}$. As in the ATLAS search, the tracks are required to have left abnormally few hits in the outer layers of the silicon tracker. A simple efficiency map based on the radial displacement is provided in the appendix of ref. [44] (table 8). As this efficiency map does not factor out the $\eta$ requirements or isolation (both of which can affect our signal models significantly), we rescale the efficiency values by an overall factor of 1.50, and impose $\eta$ acceptance and isolation separately. The rescaling factor was was determined from the effect of these cuts on our AMSB wino samples, and reliably fits with the data. The observation of 2 events with $1.4 \pm 1.2$ expected gives a $95 \%$ upper limit on a new physics signal $\times$ acceptance of $N_{95}=5.3$ events. Our modeling reproduces the exclusion contour shown in [44] very accurately for all wino lifetimes (appendix A).

As with the ATLAS disappearing track search, the fact that the stau has hard visible decay products modifies the story significantly; we once again model the impact of these decay products in $10-\mathrm{cm}$ discrete bins of radial displacement. First, a stau which decays to a hard hadronic tau very close to the interaction point can potentially provide the event's hard jet. We choose a transverse decay length of $L<2 \mathrm{~cm}$ to model this possibility simply; decay products originating further away than this are assumed to not pass the charged hadron fraction $>20 \%$ requirement placed on a jet with $p_{T}>110 \mathrm{GeV}$. Second, the stau decay products can cause the track to fail the strict $10 \mathrm{GeV}$ isolation requirement. Third, $\tilde{\tau}$ decay products alter the $E_{T}$ and can affect the jet separation requirements. As in the ATLAS search, if the $\tilde{\tau}$ yields an electron or a hadronic tau with neutral pions (hadronic tau without neutral pions), we deposit the energy of the decay products at the point where the track emanating from the center of this bin intersects a cylinder passing roughly halfway through the ECAL (HCAL) - we use $\{R, Z\}=\{155 \mathrm{~cm}, 240 \mathrm{~cm}\}(\{235 \mathrm{~cm}, 480 \mathrm{~cm}\})$ for CMS. If the stau decays within the calorimeter we deposit all of the decay product energy there; if the stau decays in the muon chamber or beyond, we assume no jet is reconstructed and all of this energy is invisible. These calorimeter deposits are labeled as jets (photons are not distinguished from jets, except by the leading jet requirement), and ${E_{T}}_{T}$, isolation, and jet separation are checked with these new objects.

Again, as in the ATLAS search, despite our very precise validation of the AMSB wino model, we stress that the additional complications due to the stau decay products greatly impact the reliability of our modeling. Because of this, we again assign a $50 \%$ modeling 


\begin{tabular}{|c|}
\hline Cut Summary of CMS displaced $e \mu$ \\
\hline Preselection \\
\hline 1 OS $e^{ \pm} \mu^{\mp}$ pair \\
$d_{\ell}>100 \mu \mathrm{m}$ \\
$p_{T, \ell}>25 \mathrm{GeV},\left|\eta_{\ell}\right|<2.5$ \\
Reject $1.44<\left|\eta_{e}\right|<1.56$ \\
$I_{\Delta R<0.3}^{\text {calo, }}<0.10, I_{\Delta R<0.4}^{\text {calo }, \mu}<0.12$ \\
$\Delta R_{\ell j}>0.5 \forall$ jets with $p_{T}>10 \mathrm{GeV}$ \\
$\Delta R_{e \mu}>0.5$ \\
$v_{T, \tilde{\ell}}<4 \mathrm{~cm}, v_{Z, \tilde{\ell}}<30 \mathrm{~cm}$ \\
Veto additional leptons \\
\hline
\end{tabular}

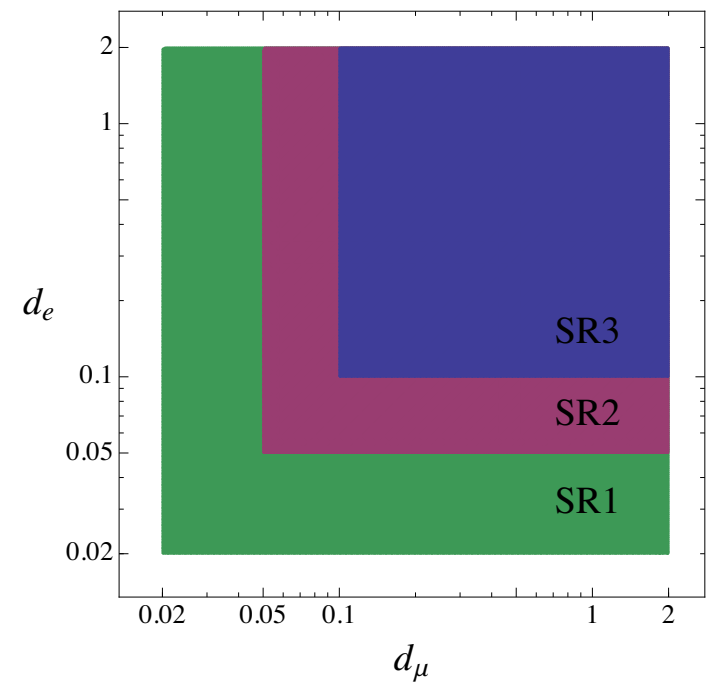

Table 2. Left: the preselection cuts used in [17] (see also [61, 62]). Right: an illustration of the cuts on the transverse impact parameter that define the three exclusive signal regions.

uncertainty in the results presented for this search. A more detailed treatment is best performed by experimentalists.

\subsection{CMS Displaced $e \mu$ search}

In the CMS displaced $e \mu$ search [17], the benchmark model considered is the direct pair production of stops that decay through small lepton-flavor-universal RPV $\lambda_{i j k}^{\prime} L_{i} Q_{j} D_{k}^{c}$ couplings $\left(\lambda_{133}^{\prime}=\lambda_{233}^{\prime}=\lambda_{333}^{\prime}\right)$ to yield displaced $\tilde{t} \rightarrow e b, \mu b$, and $\tau b$ decays. In this search, the leptons are required to be fairly hard, in the central region of the detector, and isolated from jets, other calorimeter deposits, and each other. The most distinguishing preselection requirement in the search is that the transverse impact parameter, $d_{0}$, with respect to the primary vertex is required to be larger than $100 \mu \mathrm{m}$ for both leptons. The impact parameter is actually not the point where the parent object (e.g., $\tilde{\tau}, \tau$ or $b$ ) decays, but rather the distance to the point of closest approach for the lepton's track relative to the center of the beampipe (in most other searches, the impact parameter is defined with respect to the primary vertex). This is especially important as backgrounds from $Z \rightarrow \tau \tau$ or heavy flavor tend to result in leptons that are nearly collinear with the parent due to a small mass-to-momentum ratio, and thus yield a small impact parameter even with an abnormally long-lived parent. After imposing preselection requirements, events are divided into three exclusive signal regions: SR3, where both leptons have transverse impact parameters $d_{e}$ and $d_{\mu}$ between 0.1 and $2.0 \mathrm{~cm}$; SR2, with $d_{e}$ and $d_{\mu}$ between 0.05 and $2.0 \mathrm{~cm}$, but not satisfying the requirement of SR3; and SR1, with $d_{e}$ and $d_{\mu}$ between 0.02 and $2.0 \mathrm{~cm}$, but not within SR2 or SR3. These selection requirements are summarized in table 2 .

In addition to imposing the cuts of the search, we utilize the recommended parameterization provided in [62] to model the trigger, selection, and reconstruction efficiencies for each species of lepton. We also mandate $v_{T, \tilde{\ell}}\left(v_{Z, \tilde{\ell}}\right)<4(30) \mathrm{cm}[62]$, where $v_{T, \tilde{\ell}}\left(v_{Z, \tilde{\ell}}\right)$ is 
the transverse (longitudinal) position of the secondary vertex. Beyond this range, tracking fails. To determine the 95\% CL exclusion contour, the truth-level properties of the staus and their decay products are used to derive a $c \tau$-dependent weight for each event to have the lepton transverse impact parameters falling into one of the three signal regions. ${ }^{5}$ The exclusion confidence level from the combination of the three exclusive signal region bins were derived using frequentist methods on the background estimates provided in the search and assuming the nominal NLO+NLL value for the cross-sections.

A validation against the displaced stop model considered in the CMS study is presented in appendix A. In the region of highest sensitivity, the recast agrees excellently with the results of the search. Near $c \tau \sim 1 \mathrm{~m}$ or $100 \mu \mathrm{m}$, we expect our modeling to underestimate the actual constraint slightly. We assign the recommended $25 \%$ modeling uncertainty to the search in all figures.

\subsection{Constraints on long-lived staus}

In this subsection we show the constraints on long-lived staus found from the searches described above and comment on potential avenues for improvement. To explore a wide variety of scenarios, we consider several simplified benchmark models for the pair production of $\tilde{\tau}$ NLSPs. In each model, the $\tilde{\tau}_{R}$ lifetime, $c \tau$, is treated as a free parameter (for all lifetimes of interest, the gravitino is effectively massless and has no influence on kinematics). The models considered are:

- Direct $\tilde{\tau}_{R}$ production where the $\tilde{\tau}_{R}$ is isolated at the bottom of the spectrum. $95 \%$ CL limits are shown in figure 1 (left) in the $m_{\tilde{\tau}}-c \tau$ plane. LEP2 bounds from OPAL [39] are shown in gray.

- Direct slepton production in the case where there are three nearly degenerate generations, $\tilde{e}_{R}, \tilde{\mu}_{R}$ and $\tilde{\tau}_{R}\left(m_{\tilde{e}_{R}}=m_{\tilde{\mu}_{R}}=m_{\tilde{\tau}_{R}}+10 \mathrm{GeV}\right)$ with prompt decay $\tilde{e}_{R}, \tilde{\mu}_{R} \rightarrow$ $\tilde{\tau}_{R}+\{$ soft $\} .95 \%$ CL limits are shown in figure 1 (left) in the $m_{\tilde{\tau}}-c \tau$ plane.

- Higgsino production with prompt decays $\tilde{H}^{ \pm} \rightarrow \tilde{\tau}_{R}^{ \pm} \nu$, and $\tilde{H}^{0} \rightarrow \tilde{\tau}_{R}^{ \pm} \tau^{\mp}$. 95\% CL limits are shown for $m_{\tilde{\tau}}=100$ and $300 \mathrm{GeV}$ in figure 1 (right).

- Stop production with prompt decay $\tilde{t} \rightarrow b \tilde{H}^{+} \rightarrow b \nu \tilde{\tau}_{R}^{+}$. 95\% CL limits on this scenario are shown for $m_{\tilde{\tau}}=100,300$, and $500 \mathrm{GeV}$ in figure 2 (left) with $m_{\tilde{H}}=m_{\tilde{t}}-50 \mathrm{GeV}$.

- Majorana gluino production with prompt decay $\tilde{g} \rightarrow \tilde{t} \bar{t} \rightarrow \bar{t} b \tilde{H}^{+} \rightarrow \bar{t} b \nu \tilde{\tau}_{R}^{+}$and the conjugate decay. $95 \% \mathrm{CL}$ limits are shown for $m_{\tilde{\tau}}=100,300$, and $500 \mathrm{GeV}$ in figure 2 (right) for $m_{\tilde{t}}=m_{\tilde{g}}-200 \mathrm{GeV}$ and $m_{\tilde{H}}=m_{\tilde{t}}-50 \mathrm{GeV}$.

As can be seen in figures 1 and 2, the three search strategies - HSCP, disappearing track, and displaced $e \mu-$ are complementary and constrain different lifetime regions. For

\footnotetext{
${ }^{5}$ For numerical feasibility, the finite $\tau$ lifetime of $c \tau_{\tau}=87 \mu \mathrm{m}$ was neglected. This is a small effect on the lepton impact parameter because $m_{\tau} / m_{\tilde{\tau}} \ll 1$, and thus the tau and lepton momentum are roughly collinear, i.e., $\hat{p}_{\tau} \sim \hat{p}_{\ell}$. At large $\tilde{\tau}$ lifetimes this is a very good approximation; at smaller lifetimes there can be a moderate increase in efficiency, especially for the population of SR1. This effect was verified to be $\lesssim 10 \%$.
} 

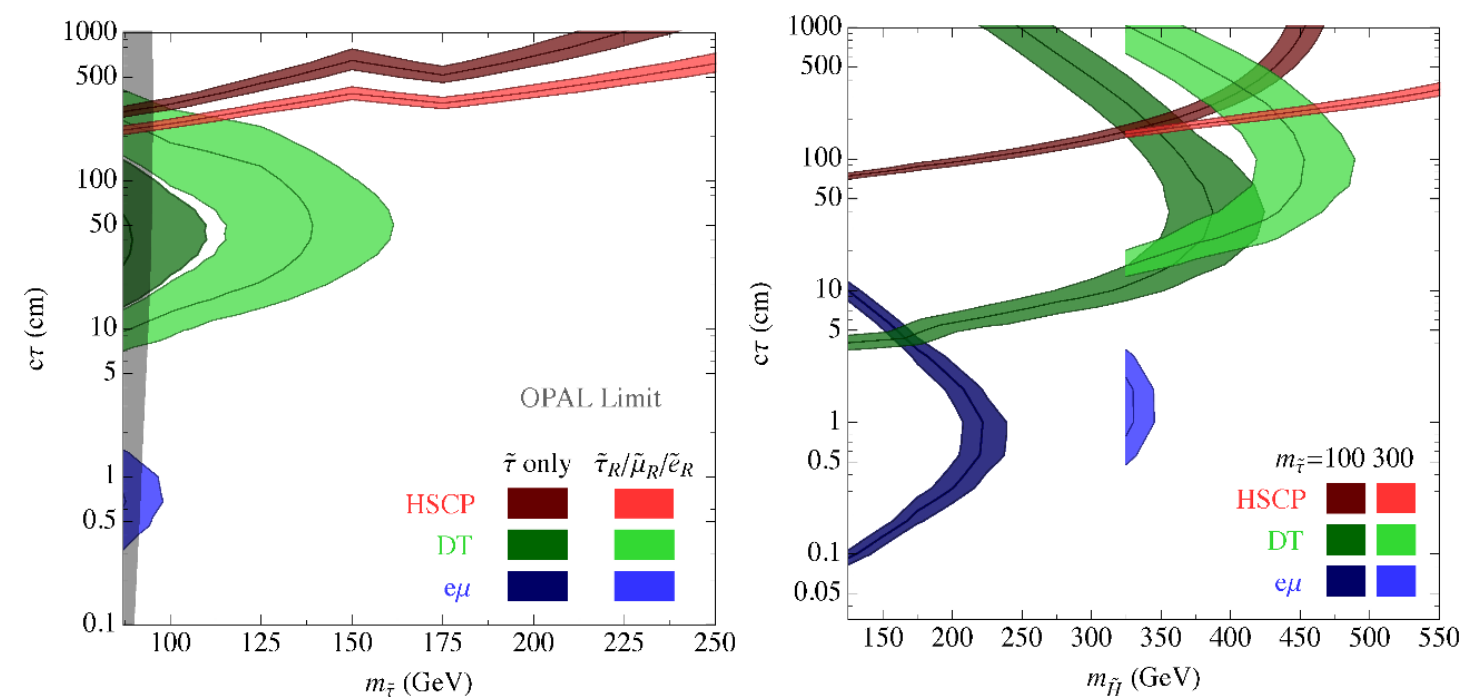

Figure 1. Left: constraints on direct production for the case of a single isolated, light, right-handed stau NLSP (dark), as well as for the case of nearly degenerate three generations of right-handed sleptons (bright). Near $c \tau \sim 1 \mathrm{~cm}$, the CMS displaced $e \mu$ search is most sensitive [17] (blue). (The $\tilde{\tau}$ only limit from this search falls well below the LEP bound and is not shown). Near $c \tau \sim 50 \mathrm{~cm}$, the disappearing track searches at CMS [44] and ATLAS [43] (green) are most sensitive; we show only the stronger of the two limits (for selected individual sensitivities, see figure 6). Above $c \tau \sim 2$ $\mathrm{m}$, the CMS heavy stable charged particle search [40] (red) sets powerful constraints. The most stringent LEP2 bounds from OPAL [39] are shown in light gray, ranging from 87 to $97 \mathrm{GeV}$. Right: constraints on production of degenerate Higgsinos decaying as $\tilde{H}^{ \pm} \rightarrow \tilde{\tau}_{R}^{ \pm} \nu / \tilde{H}_{1,2}^{0} \rightarrow \tilde{\tau}_{R}^{ \pm} \tau^{\mp}$. Only direct production of the Higgsino is used for setting a limit. A scenario with a $100 \mathrm{GeV}(300 \mathrm{GeV})$ stau is shown in dark (bright) colors. The minimum Higgsino mass shown is $125 \mathrm{GeV}$ ( $325 \mathrm{GeV}$ ). Search colors are as in figure 1 left.

the disappearing track searches, we show only the search that sets the best limit at each point, which is usually the CMS search (see figure 6 for comparison of the two disappearing track searches in a variety of scenarios). The HSCP search turns on sharply around $c \tau \sim 1$ $-3 \mathrm{~m}$, and grows stronger at longer lifetimes. The disappearing track searches are most sensitive in the $c \tau \sim 50 \mathrm{~cm}$ range. The displaced $e \mu$ search peaks around $c \tau \sim 1 \mathrm{~cm}$.

As the HSCP search is powerful and has a very high acceptance, we have no suggestions for potential improvements to that search, short of providing efficiency maps for the trackeronly search to enhance the usability of the results. We note that in cascade decay scenarios with a large mass hierarchy between the initially produced parent particle (e.g., a gluino) and the stau, the requirement that $\beta$ differ from $c$ typically fails above a certain parent particle mass. Of course, in most of these scenarios direct production of the stau would be constrained by the HSCP search.

The disappearing track searches use very different selections and methods between the two experiments. The CMS search has very high background rejection, resulting in only two events in their signal region, while the ATLAS search admits dozens of background events with a more inclusive selection. Between the two searches, CMS typically sets the stronger limit; however, at shorter lifetimes the two are nearly identical in strength. 

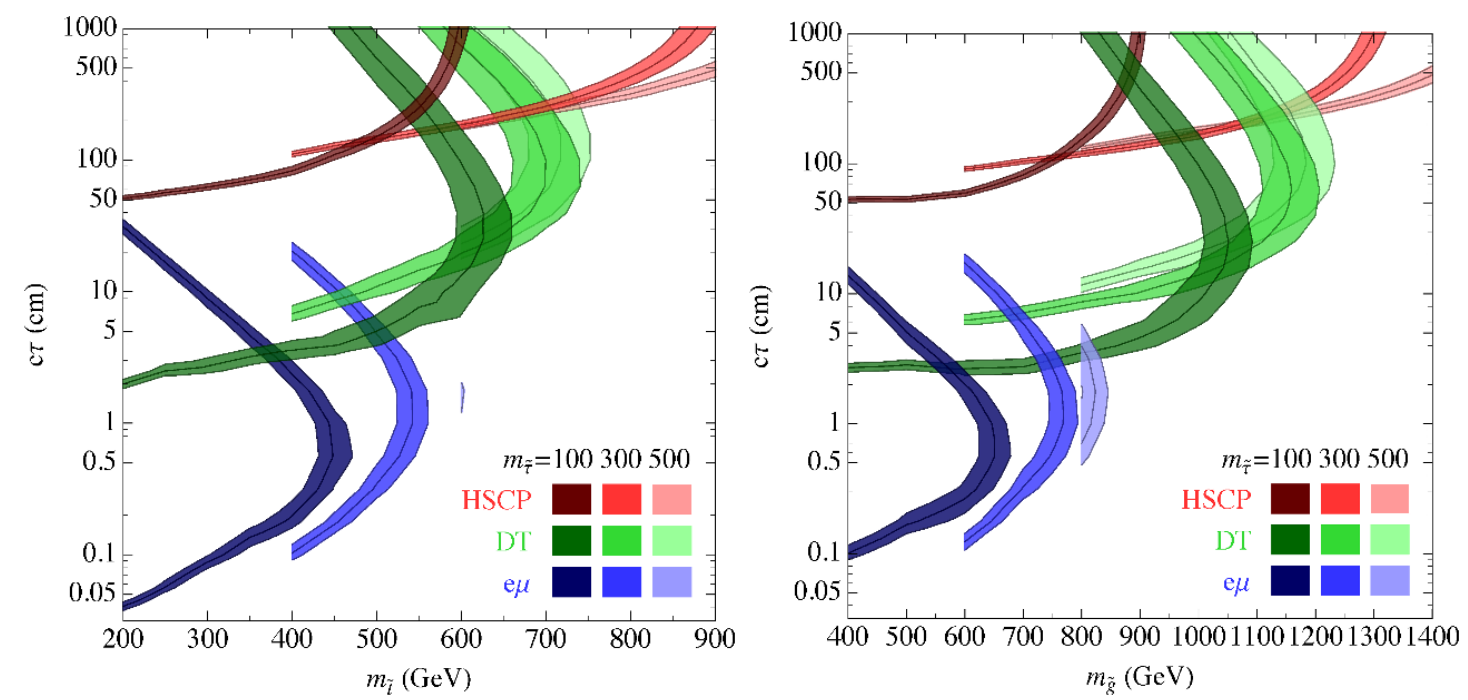

Figure 2. Left: constraints on production of right-handed stops decaying as $\tilde{t} \rightarrow b \tilde{H}^{+} \rightarrow b \nu \tilde{\tau}_{R}^{+}$ with $m_{\tilde{H}^{+}}=m_{\tilde{t}}-50 \mathrm{GeV}$. Only the direct production of stops is used for setting a limit. A scenario with a $100(300$ [500]) GeV stau is shown in dark (bright [light]) colors. The minimum stop mass shown is 200 (400 [600]) GeV. Search colors are as in figure 1 left. Right: constraints on production of Majorana gluinos decaying through stops and Higgsinos into a displaced stau final state. Only the direct production of the gluinos is used to set a limit. All coloration is as in the stop model figure 2 left. The minimum gluino mass shown is 400 (600 [800]) GeV. Dirac gluinos [63], which only give opposite-sign leptons, have a cross-section $\times$ efficiency that is four times larger than the results presented here.

Part of the reason that CMS is able to set stronger limits at higher $c \tau$ is that ATLAS vetoes events with activity in the muon chamber, thus losing a large fraction of events that effectively contain a heavy stable charged particle, which are very well constrained by the HSCP searches. See appendix A for more details and plots comparing the two searches. The disappearing track searches peak at a proper lifetime of around 20 to 50 $\mathrm{cm}$. These searches are not able to constrain direct production of $\tilde{\tau}_{R}$ beyond the limits set by OPAL, but production of three near-degenerate species of right-handed sleptons are constrained up to $\sim 140 \mathrm{GeV}$. Higgsinos are constrained by disappearing track searches up to $375-450 \mathrm{GeV}$, stops are constrained up to $600-700 \mathrm{GeV}$, and gluinos are constrained up to $1050-1200 \mathrm{GeV}$.

Although modeling signal acceptance in these searches is challenging, modeling the background for the searches is substantially more difficult, so while we have some suggestions for modifications to these searches that would enhance sensitivity to displaced slepton decays, we stress that we cannot quantitatively assess how these modifications will affect the backgrounds. Thus our most important suggestion for both experiments is to simply include the NLSP $\tilde{\tau}_{R}$ benchmark model in the search.

For the ATLAS disappearing track search [43], the pointing and timing capabilities of the ATLAS ECAL [64] could allow for ECAL deposits originating away from the IP and/or arriving later than the rest of the calorimeter activity (due to the slower speed 
of the staus and decay geometry) to be distinguished from prompt jets in the search. More importantly, these capabilities could potentially allow for an additional discriminant to improve sensitivity to staus, i.e., substantial, late-time calorimeter deposits that point toward the vicinity of where the disappearing track vanished. If computationally feasible, the pointing information could even be utilized as a constraint to facilitate an off-line reconstruction of the kinked track in the TRT. Using an additional discriminant of this kind could allow for a relaxation of the harsh $p_{T}$ cuts while maintaining, if not improving, background rejection. We additionally stress that providing efficiency maps would be invaluable for recasting.

For the CMS disappearing track search [44], the very strict isolation cut on the track, $E_{\text {calo }}^{\Delta R<0.5}<10 \mathrm{GeV}$, significantly reduces sensitivity to staus as the stau decay products often fall within this cone (and do so more frequently at larger displacements). As the CMS ECAL timing resolution is very good [65], energy deposits within the isolation cone that arrive later than expected could be dropped from $E_{\text {calo }}^{\Delta R<0.5}$. The basic preselection of this search with an added off-line kinked track requirement in place of the stringent isolation requirement could provide background rejection, but as the analysis techniques are very different, this may be regarded as a distinct search proposal.

In the $c \tau \sim 1 \mathrm{~cm}$ regime best covered by the CMS displaced $e \mu$ search, there are no limits on the direct production of staus. Including the production of nearly degenerate $\tilde{\mu}_{R}$ and $\tilde{e}_{R}$ increases the overall production cross-section enough to yield mild constraints in a narrow lifetime window, but still below those set by OPAL. For Higgsino-, stop-, and gluino-initiated $\tilde{\tau}_{R}$ production, the reach extends to $225-325 \mathrm{GeV}, 450-600 \mathrm{GeV}$, and $650-$ $800 \mathrm{GeV}$ for lifetimes of $\mathcal{O}(1 \mathrm{~cm})$, with sensitivity dying off for longer and shorter lifetimes.

As the CMS displaced $e \mu$ search uses the most recently designed strategy of the four searches, it is not surprising that this is where we found the most potential for improvement. Although much of the behavior of the sensitivities shown in figures $1 \& 2$ is a straightforward result of the falling production cross-section with mass and the experimentally available window for lepton impact parameters, there are several other factors in play that influence these results. Here, we highlight several important points:

- In GMSB, the NLSP is typically a right-handed stau, which decays to a highly righthanded polarized $\tau$. This is important because the tau polarization significantly affects the energy of the final state light lepton [66, 67]. The differential lepton energy distribution from a polarized stau decay can be written as [68]

$$
\frac{1}{\Gamma} \frac{d \Gamma}{d x}=\frac{2}{3}\left[5-36 x^{2}(1-x)+P_{\tilde{\tau}}\left(1-36 x^{2}+64 x^{3}\right)\right],
$$

where $x \equiv E_{\ell} / m_{\tilde{\tau}}, P_{\tilde{\tau}}$ is $+1(-1)$ for right-handed (left-handed) staus, and we have neglected corrections of order $\left(m_{\ell} / m_{\tau}\right)^{2}$ and $m_{\tau} / m_{\tilde{\tau}}$. Right-handed staus tend to suppress the energy given to the light lepton, while left-handed staus enhance it (see figure 3). In the rest frame of a pure right-handed stau, about $50 \%$ of decays impart less than $13 \%$ of the stau energy to the light lepton. As the CMS search requires relatively hard leptons with $p_{T, \ell}>25 \mathrm{GeV}$, this preference for soft leptons greatly degrades acceptance, especially for lighter staus. Lowering the $p_{T}$ threshold for one or 


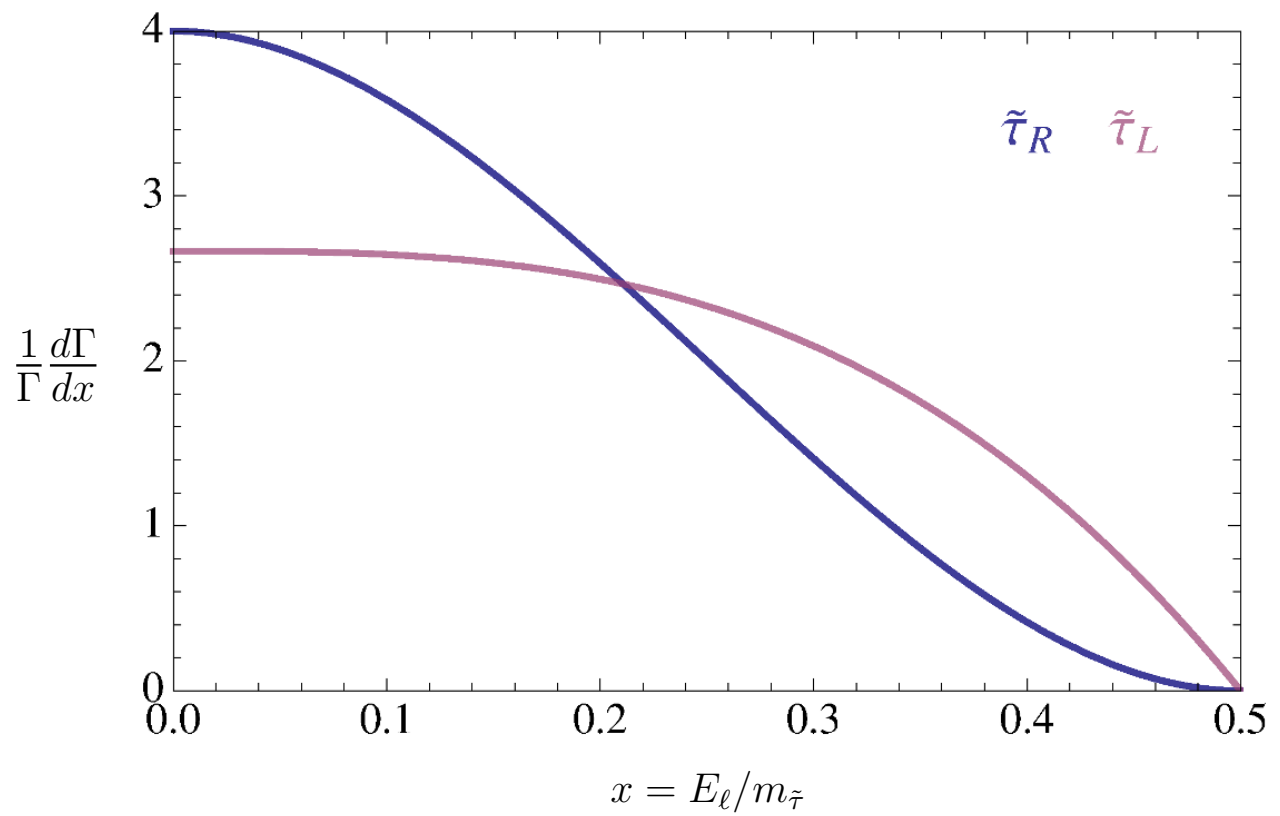

Figure 3. Distribution of lepton energies from stau decays (neglecting terms of order $\left(m_{\ell} / m_{\tau}\right)^{2}$ and $\left.m_{\tau} / m_{\tilde{\tau}}\right)$. Note how heavily preferred soft leptons are for right-handed staus.

both species of leptons would greatly increase the acceptance for $\tilde{\tau}_{R}$ NLSPs. While lowering lepton $p_{T}$ thresholds may present difficulties for triggering on direct stau production, it can make a significant difference in benchmark models where the stau is produced at the bottom of a cascade decay and other hard objects are available for triggering.

- The presence of Majorana particles in both the gluino and Higgsino simplified models results in same-sign leptons roughly $50 \%$ of the time. ${ }^{6}$ As the displaced $e \mu$ search requires opposite-sign leptons, sensitivity to these scenarios is trivially degraded. In principle, data-driven techniques that utilize the same-sign displaced lepton background to predict the backgrounds in the opposite-sign regions could wash out a signal (such as the method used in the CMS displaced $e \mu$ search for determining the heavy flavor backgrounds). While especially dangerous for gluinos and Higgsinos, this control region contamination can even happen in the LQD stop model considered in the CMS displaced $e \mu$ search, where the long lifetimes make mesino oscillation of the stops [69] a viable possibility, potentially leading to as many as 3 in 8 events possessing leptons with the same sign [70].

- In both the cases of gluino and Higgsino production, many of the events contain additional leptons (from the decays of either tops or taus) that are vetoed in the search. The prevalence of additional prompt leptons depends on the particular production and decay modes in a given simplified model, but, aside from the case of direct pro-

\footnotetext{
${ }^{6}$ In the Higgsino case, the fraction of the events containing same-sign leptons is less than $50 \%$, since $\sim 20 \%$ of the total production rate comes from $\tilde{\chi}^{+} \tilde{\chi}^{-}$production.
} 
duction, additional leptons are a generic possibility. In the gluino benchmark model considered here, nearly half of all signal events are discarded due to the presence of an additional prompt lepton. Importantly, this veto should be unnecessary, as no major backgrounds tend to be produced with additional isolated leptons at any appreciable rate. In most models of interest, an approximate $\mathbf{Z}_{2}$ symmetry is what provides the displacement. Thus, typically only two genuinely displaced leptons will appear per event, and combinatoric ambiguities can be resolved simply by choosing the leptons with the highest impact parameters.

- The CMS displaced $e \mu$ search uses very tight isolation requirements to improve rejection of heavy flavor backgrounds. These isolation requirements are significant enough that the hadronic activity is sufficient to reduce the overall efficiency by $10-15 \%$ in the case of direct stau production, and $25-35 \%$ in the gluino case where there are many additional jets. In large part, this is another side effect of the low lepton $p_{T}$ arising from right-handed polarized $\tau$ decays — softer leptons require less hadronic activity to fail isolation requirements. At larger transverse displacements, the heavy flavor background is greatly reduced, so looser isolation criteria, particularly in SR3, would serve to enhance sensitivity, especially for longer lifetimes.

\section{Models with displaced same-flavor leptons}

Although a search for an opposite-sign $e$ and $\mu$ with large impact parameters is in principle sensitive to displaced stau NLSPs, and more generally to any new physics that gives rise to displaced decays exhibiting lepton-flavor universality (e.g., displaced $\tilde{\chi}^{+} \rightarrow W^{+} \tilde{G}$ ), it is insensitive to models that have displaced same-flavor leptons originating from different vertices. Even the RPV stop benchmark model considered in the CMS displaced $e \mu$ search [17] would more generically result in a same-flavor signature. In the CMS search, it was assumed that the $L Q D$ RPV operators were lepton-flavor-universal, but, as the known superpotential couplings (i.e., SM Yukawas) exhibit large hierarchies, this assumption is not well-motivated. Hierarchical couplings would generically produce one dominant stop decay path. Due to the additional hadronic activity at these displaced vertices, this RPV stop model is powerfully constrained by other displaced searches [11, 15, 71], and will not be discussed in more detail in this work.

Rather generally, one can frame displaced lepton models as a charged particle $\phi^{ \pm}$that decays into an invisible particle $\chi$ and one of the three flavors of charged leptons, $e, \mu$, or $\tau$ with branching fractions, $B_{e}, B_{\mu}, B_{\tau}$, respectively, that sum to unity. If one has that $B_{\tau}=0$ and $B_{e} B_{\mu}<0.06$, then this model has fewer $e^{ \pm} \mu^{\mp}$ events than the analogous $B_{\tau}=1$ case. Similarly, if one has $B_{e}=0$ and $B_{\mu}>B_{\tau}$, there are, again fewer $e^{ \pm} \mu^{\mp}$ events. Of course, these oversimplifications neglect the softer charged leptons arising in $\tau$ decays and the important effects of $\tau$ helicity. For simplicity of discussion, we will use the requirements,

$$
B_{e} B_{\mu}<0.01 \text { and } B_{\tau} \lesssim 0.1
$$


to illustrate the parametric requirements for being at most weakly constrained by the CMS displaced $e \mu$ search, i.e., at a level below the lepton-flavor-universal $\tilde{\tau}$ NLSP models in section 2. These conditions are pointed out to highlight the regions of parameter space where a same-flavor search is essential, as there is little hope that a displaced $e \mu$ search alone will be able to constrain that scenario. We stress that even when these conditions are maximally violated, the search we will propose in section 4 will generically set limits which, at the very least, would be competitive with [17] and will be more sensitive across large regions of parameter space. The following subsections will discuss in detail several models that preferentially give rise to pairs of displaced single muons; the extension to electrons is trivial.

\subsection{Slepton Co-NLSP}

A realistic, minimal possibility within GMSB is that the right-handed sleptons are all co-NLSPs [47], each decaying to their respective SM partner. In this case, the sameflavor muon and same-flavor electron signal will appear together, along with the weakly constrained tau signal. The proposed same-flavor search discussed in the next section would be the best handle on long-lived slepton co-NLSPs, even without invoking one of the mechanisms discussed below to produce same-flavor dominated signatures.

\subsection{Sleptons in extended gauge mediation}

Models of extended gauge mediation (EGMSB) [72], where one introduces direct couplings into the superpotential between the SM and messenger superfields, provide a simple mechanism whereby a first- or second-generation slepton can become the NLSP [73, 74]. In order to not flood the casual reader with technical details, here we only present a streamlined discussion. Further details are provided in appendix B.

For EGMSB to directly affect right-handed sleptons, one must introduce couplings in the superpotential of the form

$$
W \supset \kappa_{i} E_{i}^{c} \Phi \tilde{\Phi}
$$

where $\Phi, \tilde{\Phi}$ are messengers with appropriate gauge quantum numbers. For simplicity, we will focus on a model where $W \supset \kappa_{i} E_{i}^{c} \Phi_{U} \Phi_{\bar{D}}$, where $\Phi_{U}$ and $\Phi_{\bar{D}}$ have the quantum numbers $(\overline{3}, 1)_{-\frac{2}{3}}$ and $(3,1)_{-\frac{1}{3}}$, respectively. With the general formulas from [24], the EGMSB contribution to the slepton mass can be determined. This EGMSB-induced splitting can be written (neglecting the effects of running) as a function of $m_{\tilde{\ell}}$ and $\kappa_{i}$,

$$
\Delta m_{\tilde{\mu}} \sim 25 \kappa_{2}^{2} m_{\tilde{\ell}}
$$

for $\kappa_{1}, \kappa_{3} \ll \kappa_{2}$ and $\Delta m_{\tilde{\mu}} \ll m_{\tilde{\ell}}$; see appendix B for details. With $\kappa_{2} \sim 6 \times 10^{-2}$, this will cause an $\mathcal{O}(10 \mathrm{GeV})$ splitting between the smuon and the other right-handed sleptons.

In order to preferentially generate same-flavor final states, some alignment is required. The lightest slepton eigenvalue points in the $\vec{\kappa}$ direction within flavor space, so the branching ratios are simply $B_{i}=\kappa_{i}^{2} /\left(\kappa_{1}^{2}+\kappa_{2}^{2}+\kappa_{3}^{2}\right)$. From our simple conditions (3.1), we can infer that

$$
\frac{\kappa_{1}}{\kappa_{2}} \ll 0.1 \text { and } \frac{\kappa_{3}}{\kappa_{2}} \lesssim 0.3
$$


forces us into a region of parameter space where a displaced same-flavor search is essential to constrain this scenario.

As this simple model exhibits rank one chiral flavor violation [75] in the right-handed sector, it is insulated against many flavor constraints as compared to an anarchic scenario. The most constraining flavor observable on the right-handed slepton mass matrix is $\mu \rightarrow$ er [76], which typically constrains the product $\kappa_{1} \kappa_{2} \lesssim 10^{-3}[77,78]$. For larger splittings and/or particular choices of EGMSB couplings (e.g., B.10), $\mu \rightarrow e \gamma$ could reduce the viable parameter space, but any model with a pure $\tilde{\mu}$ or $\tilde{e}$ NLSP will be safe from this constraint. A general study of flavor constraints in leptonic $\chi \mathrm{FV}$ is well beyond the scope of this work.

To summarize, EGMSB models can produce a same-flavor signature by splitting the $\tilde{e}$ or $\tilde{\mu}$ from the other sleptons using a fairly small, $\mathcal{O}\left(10^{-1}\right)$, EGMSB coupling. The simplified model requires only a moderate alignment of the flavored coupling $\vec{\kappa}$ with the electron $\left(\kappa_{1}\right)$ or muon $\left(\kappa_{2}\right)$ direction in order to avoid flavor constraints and give a relatively pure displaced $e^{+} e^{-}$or $\mu^{+} \mu^{-}$signal. In principle, this slepton splitting mechanism is modular and could be combined with other EGMSB operators, e.g., to alleviate tuning in the Higgs sector.

\section{3 $\quad R$-parity violating decays of staus via LLE operators}

Another model generically predicting flavor-non-universal slepton decays arises in the presence of R-parity-violating LLE operators [3]. With $R$-parity violated, the following trilinear superpotential terms are now allowed:

$$
W \ni \lambda_{i j k} L_{i} L_{j} E_{k}^{c}+\lambda_{i j k}^{\prime} L_{i} Q_{j} D_{k}^{c}+\lambda_{i j k}^{\prime \prime} U_{i}^{c} D_{j}^{c} D_{k}^{c} .
$$

A stau that is at least partially left-handed can decay via the $\lambda_{i 32} L_{i} L_{3} E_{2}^{c}$ operator to give a pure muon final state, i.e., $\tilde{\tau}_{1}^{+} \rightarrow \mu^{+} \nu_{i}$. The stau lifetime in these models can be expressed as

$$
c \tau \approx 1 \mathrm{~cm}\left(\frac{10^{-7}}{\lambda_{i 32}}\right)^{2}\left(\frac{100 \mathrm{GeV}}{m_{\tilde{\tau}}}\right) \sec ^{2} \theta_{\tilde{\tau}},
$$

where the mixing angle $\theta_{\tilde{\tau}}=0$ corresponds to a pure left-handed state, and we have defined $\lambda_{i 32} \equiv \sqrt{\lambda_{132}^{2}+\lambda_{232}^{2}}$.

From a model-building perspective, it is more generic to have an (N)LSP stau be righthanded than left-handed, but some models, such as GGM [79], can readily accommodate a spectrum with an NSLP left-handed stau. However, some degree of left-right mixing is generic, due to the Yukawa-induced mass term, $\left(\mu^{*} m_{\tau} \tan \beta\right) \tilde{\tau}_{L} \tilde{\tau}_{R}$. If the $\lambda_{i 32}$ coupling is the only non-zero RPV coupling, then the stau decay will proceed to the $100 \%$ muon final state, even for $\theta_{\tilde{\tau}} \sim \pi / 2$. More generally, from the criteria of (3.1), the $\lambda_{i 32}$ coupling must dominate by

$$
\begin{aligned}
& \lambda_{i 32} \gg 10 \sqrt{\left(\lambda_{123}^{2}+\lambda_{133}^{2}\right) \tan ^{2} \theta_{\tilde{\tau}}+\lambda_{131}^{2}+\lambda_{231}^{2}} \\
& \lambda_{i 32} \gtrsim 3 \sqrt{\lambda_{233}^{2}\left(\tan ^{2} \theta_{\tilde{\tau}}+2\right)+\lambda_{133}^{2}}
\end{aligned}
$$

in order for an opposite-flavor search to have little to no sensitivity. Additionally, the LQD couplings $\lambda_{3 i j}^{\prime}$ must also be small to evade constraints from the displaced jet searches $[15$, 
71]. Since the small RPV couplings yield long lifetimes, we note that $\tilde{\nu} \rightarrow \tilde{\tau}+\{$ soft $\}$ transitions will occur much more rapidly than $\tilde{\nu} \rightarrow \ell^{+} \ell^{\prime-}$ decays, unless $m_{\tilde{\nu}}-m_{\tilde{\tau}}<$ $1 \mathrm{GeV}[80]$.

There are typically no flavor constraints in this model due to the small sizes of the RPV couplings; the only exception is proton decay when UDD operators are simultaneously introduced. For particular flavor structures, some of these bounds require that $\left|\lambda_{i j k} \lambda_{i^{\prime} j^{\prime} k^{\prime}}^{\prime \prime}\right| \lesssim 10^{-26}[3]$. Imposing baryon number conservation removes all such issues. Alternatively, as long as first-generation particles are not heavily involved, UDD coefficients could still be as large as $10^{-3}$ without any conflict with proton decay constraints [3]. Amusingly, it would be possible for displaced lepton signatures to live alongside a prompt paired-dijet signature of RPV stops.

\subsection{Lepton-flavored dark matter from freezein}

Models of flavored dark matter, where the dark matter is charged under the flavor symmetries of either the quarks or the leptons, can give rise to novel signatures at the LHC [81]. In these models, the lifetime of the decaying particle is generically directly related to the cosmological abundance of dark matter. Long lifetimes at colliders require couplings which are typically much smaller than those required for DM to originate from thermal freezeout to the SM. On the other hand, very small couplings are naturally predicted by models of freezein, where dark matter is produced by the out-of-equilibrium decays of a particle in the thermal bath [82].

Minimal models of lepton-flavored freezein DM can be written in terms of a fermionic DM flavor multiplet $\chi_{i}$ and a charged scalar $\zeta$,

$$
\mathcal{L} \supset y_{i j}^{L D M} \ell_{i}^{c} \zeta^{-} \chi_{i}+m_{\chi, i j} \chi_{i} \bar{\chi}_{j}+\text { h.c. }+m_{\zeta}^{2} \zeta^{+} \zeta^{-},
$$

or a scalar DM flavor multiplet $S_{i}$ and a charged fermion $\psi$,

$$
\mathcal{L} \supset y_{i j}^{L D M} \ell_{i}^{c} \psi S_{j}+m_{\psi} \psi \bar{\psi}+\text { h.c. }+m_{S, i j}^{2} S_{i}^{\dagger} S_{j} .
$$

The charged particle is present in the thermal bath, and decays via the small flavored Yukawa coupling $y_{i j}^{L D M}$. When $y \lll 1$, the resulting out-of-equilibrium decays produce a relic abundance of DM that is directly proportional to the width of the charged parent. Note that unlike all other models discussed in this work so far, the new charged particle of the model in (3.10), $\psi$, is a fermion, and thus has a higher production cross-section for a given mass. For this reason we will specialize to that model throughout the rest of this subsection. For freezein in a standard thermal cosmology, the values of $y$ that yield acceptable relic abundances imply that $\psi$ will be detector-stable, unless the dark matter mass is low enough to present serious issues with structure formation, i.e., free-streaming and Tremaine-Gunn constraints [83, 84]. However, an alternative generic possibility is that, at the time of dark matter freezein, characterized by the temperature $T_{F I} \sim m_{\psi} / 4$, the energy density of the universe is dominated by the coherent oscillations of a massive field, e.g., an inflaton or a heavy modulus $[85,86]$. This non-thermal phase of evolution terminates when the massive field decays to radiation (e.g., the SM). Some non-thermal 
epoch is required in any inflationary cosmology in order to populate the thermal bath of the SM after inflation, and indeed, the prime example of such an epoch is post-inflationary reheating. The coupling strengths necessary for freezein during a matter-dominated era to produce the correct DM relic abundance today are much larger than the couplings required by standard radiation-dominated freezein, as the higher initial DM density is diluted by the entropy released when the heavy particle decays. These coupling strengths can provide lifetimes relevant for displaced decays at colliders [87]. We discuss the details of this mechanism in appendix $\mathrm{C}$.

For the purposes of this work, we will use a simple model with

$$
\mathcal{L} \supset y_{i} \ell_{i}^{c} \psi S+m_{\psi} \psi \bar{\psi}+\text { h.c. }+m_{S}^{2} S^{\dagger} S
$$

where the Yukawa coupling $y_{i}$ is flavor-aligned with one species of lepton. For a lifetime $c \tau$ yielding a displaced collider signatures and mass $m_{\psi}$, one can typically choose $m_{S}$ and $T_{\mathrm{RH}}$ such that the dark matter relic abundance matches the observed value today (see appendix C). For simplicity of illustration, we will always choose the dark matter to be effectively massless for the purposes of LHC kinematics, i.e., $m_{S} \ll m_{\psi}$, although in some instances (lower $\psi$ mass and/or shorter lifetimes) this may imply that the $S$ relic abundance represents only a portion of the dark matter density today.

As $y_{i} \lesssim 10^{-7}$, there are no constraints from precision flavor observables. From our conditions (3.1), we can infer that

$$
\frac{y_{1}}{y_{2}} \ll 0.1 \text { and } \frac{y_{3}}{y_{2}} \lesssim 0.3
$$

forces us into a region of parameter space where a displaced same-flavor search is essential to constrain this scenario.

\section{A search for displaced same-flavor leptons}

In this section, we will construct a simple search for same-flavor leptons with large impact parameters. The heavy stable charged particle searches have been projected to $13 \mathrm{TeV}$ elsewhere [88], and while there are new results using $2.4 \mathrm{fb}^{-1}$ of data at $13 \mathrm{TeV}$ [89], we will not recast these in this work. While we have made some suggestions on how to improve the sensitivity of the existing disappearing track searches to this kinked track scenario, estimating backgrounds for these searches is beyond the scope of this work, so we will make no attempt to design $13 \mathrm{TeV}$ versions of these searches.

Estimating the backgrounds to a search for displaced same-flavor leptons is challenging, and, especially in the case of leptons coming from heavy flavor, requires data-driven techniques. To approximate the backgrounds at $13 \mathrm{TeV}$ for a displaced same-flavor lepton search, we will utilize the CMS displaced $e \mu$ search's $8 \mathrm{TeV}$ background projections, shown in figure 1 of ref. [17], which are in very good agreement with the data. In order to use these backgrounds directly, we will mirror the cuts of the CMS displaced $e \mu$ search (table 2), adding a $0.3<\Delta \phi_{\mu \mu}<2.8$ cut to remove backgrounds from cosmic muons and cosmic 
muon bundles (we assume this has a negligible effect on all other backgrounds ${ }^{7}$ ). While existing Run II studies (e.g., [90]) rely on lepton triggers with $p_{T}$ thresholds well below the lepton acceptance cuts of [17], these thresholds will almost certainly increase with higher luminosity. For the purpose of this sensitivity study, we choose to continue with the Run I cuts, instead of confronting backgrounds we cannot reliably estimate.

There are several backgrounds relevant for the $e \mu$ channel [17]: heavy flavor, $Z \rightarrow \tau \tau$, top, and other electroweak processes. As all of these backgrounds can contain $b \mathrm{~s}, c \mathrm{~s}$, and/or $\tau$ s which can give a genuine displacement due to their long lifetimes, it is a priori unclear what fraction of the background has a genuinely large lepton impact parameter and what fraction is due to track mis-reconstruction or detector effects creating an artificial displacement from prompt leptons. As both $t \bar{t}$ and electroweak backgrounds are very small in the $e \mu$ search, we assume that prompt sources of same-flavor leptons, notably $Z \rightarrow \ell^{+} \ell^{-}$, can be neglected or controlled, e.g., by cutting out a $Z$ window in the lepton invariant mass.

Estimating the $8 \mathrm{TeV}$ same-flavor backgrounds from the data presented in the CMS opposite-flavor search (figure 1 of ref. [17]) requires several assumptions and approximations. First, we assume that, for each background $x$, the two lepton displacements are uncorrelated and the population of background events can be factorized, i.e.,

$$
\begin{aligned}
P_{e \mu}^{x}\left(d_{e}, d_{\mu}\right) & =2 P_{e}^{x}\left(d_{e}\right) P_{\mu}^{x}\left(d_{\mu}\right) \\
P_{e e}^{x}\left(d_{e_{1}}, d_{e_{2}}\right) & =P_{e}^{x}\left(d_{e_{1}}\right) P_{e}^{x}\left(d_{e_{2}}\right) \\
P_{\mu \mu}^{x}\left(d_{\mu_{1}}, d_{\mu_{2}}\right) & =P_{\mu}^{x}\left(d_{\mu_{1}}\right) P_{\mu}^{x}\left(d_{\mu_{2}}\right) .
\end{aligned}
$$

We also assume that prior to the application of displacement cuts and selection efficiencies [62], all backgrounds are flavor universal. Guided by the assumption that genuine displacement of $b \mathrm{~s}, c \mathrm{~s}$, or $\tau$ parents dominate the backgrounds at smaller displacement, we assume that the background shape in the first several bins can be fit as

$$
P_{\ell}^{x}(d)=\epsilon_{\ell}(d) A_{\ell}^{x} e^{-\alpha_{\ell}^{x} d}
$$

where $\epsilon_{\ell}$ is the displacement-dependent lepton selection efficiency [62], and the fit parameters $A_{\ell}^{x}$ and $\alpha_{\ell}^{x}$ for each background, $x$, depend only on the lepton species. This exponential assumption is supported by the data in figure 1 of ref. [17]. However, due to the preselection requirement of $d_{\ell}>0.1 \mathrm{~mm}$, the $d_{\ell} \leq 0.1 \mathrm{~mm}$ data is not presented at all in the search. In order to approximate these regions, we use the first four $d_{0}$ bins to derive $\alpha_{\ell}^{x}$ in (4.4) for each of the three main backgrounds $x$ (Z, HF and top). ${ }^{8}$ With this exponential we can extrapolate an expression for the missing $0 \leq d_{0} \leq 0.1 \mathrm{~mm}$ bin in each background for both electron and muon samples (when the other lepton has $d_{0}>0.1$ $\mathrm{mm})$. Then, using the ABCD method across the selections $d_{0} \leq 0.1 \mathrm{~mm}$ and $d_{0}>0.1$ $\mathrm{mm}$ for both electrons and muons, we can estimate the $e$ and $\mu d_{0} \leq 0.1 \mathrm{~mm}$ bin sepa-

\footnotetext{
${ }^{7}$ In fact, this is a conservative assumption, as the $\Delta \phi$ cut could potentially help suppress heavy flavor and $Z \rightarrow \tau \tau$ backgrounds even further, at minimal cost to signal acceptance.

${ }^{8}$ For simplicity, we neglect the negligibly small "other EW" backgrounds. When we extrapolate to $13 \mathrm{TeV}$, we assume these backgrounds remain negligibly small.
} 


\begin{tabular}{|l|ccc|}
\hline Sample & SR1 & SR2 & SR3 \\
\hline$e^{ \pm} \mu^{\mp} 8 \mathrm{TeV}$ (CMS actual) & $18.0 \pm 3.8$ & $1.01 \pm 0.31$ & $0.051 \pm 0.018$ \\
$e^{ \pm} \mu^{\mp} 8 \mathrm{TeV}$ (our estimate) & $19.8 \pm 4.1$ & $0.92 \pm 0.28$ & $0.055 \pm 0.024$ \\
\hline$e^{ \pm} \mu^{\mp} 13 \mathrm{TeV}$ & $34.1 \pm 6.5$ & $1.49 \pm 0.44$ & $0.086 \pm 0.038$ \\
$e^{+} e^{-} 13 \mathrm{TeV}$ & $25.2 \pm 3.6$ & $1.43 \pm 0.33$ & $0.31 \pm 0.06$ \\
$\mu^{+} \mu^{-} 13 \mathrm{TeV}$ & $13.0 \pm 3.1$ & $0.50 \pm 0.15$ & $0.012 \pm 0.006$ \\
\hline
\end{tabular}

Table 3. Projected backgrounds estimated using the methods described in the text. Our $13 \mathrm{TeV}$ extrapolations assume $20 \mathrm{fb}^{-1}$, a $30 \%$ systematic uncertainty on the heavy flavor backgrounds, and $10 \%$ systematic uncertainties on all other backgrounds.

rately for each of the three background channels. ${ }^{9}$ Using this information and factoring out the identification efficiencies, we can derive normalizations $A_{e}^{x}$ and $A_{\mu}^{x}$ in (4.4) for the full distributions under the assumption that the total truth-level background events are flavor-universal (i.e., the same number of electrons and muons are found in each sample). With this factor, we normalize the CMS background distributions in figure 1 of ref. [17] and, using these normalized distributions as the $P_{\ell}^{x}(d)$ in (4.1), have enough information to make an estimate of the $8 \mathrm{TeV}$ same-flavor backgrounds in the signal regions of the CMS search. We apply a systematic uncertainty of $30 \%$ (10\%) to our estimates of the HF ( $Z$ and top) backgrounds. As a cross-check, we compare our resulting estimate for the $8 \mathrm{TeV}$ e $\mu$ backgrounds to the published background estimates in table 3. Our estimates agree with the expected experimental backgrounds to within $10 \%$ of the published results. The residual disagreement, which is too small to substantially affect our conclusions, can be understood as a breakdown of our assumption that the two lepton displacements are uncorrelated.

To project these background estimates to $13 \mathrm{TeV}$, we again must make several assumptions and approximations. For top and $Z$ backgrounds, we assume these are dominated by near-threshold production, so we simply rescale these by the ratio of the inclusive crosssections, $\sigma_{X}(13 \mathrm{TeV}) / \sigma_{X}(8 \mathrm{TeV})$, and neglect effects of altered lepton kinematics. This cross-section ratio is 3.28 for top and 1.74 for $Z$ production [91]. At $8 \mathrm{TeV}$ the HF backgrounds are dominant in all signal regions, and at $13 \mathrm{TeV}$ we expect this to remain true. However, there are multiple competing effects that can influence the scaling of the HF background. First, the $b \bar{b}$ cross-section rises by 1.53 (we do not separately model the charm contribution for simplicity) [91]. Additionally, the $b \bar{b}$ kinematics change so that more $b \mathrm{~s}$ are boosted. Boosted $b$ s produce harder leptons and survive to longer displacements before decaying, but also result in leptons with smaller opening angles and produce harder hadrons that can foil isolation. Whether more boost of the parent $B$ meson translates to more isolated displaced leptons is unclear a priori. In Monte Carlo $b \bar{b}$ samples, we ex-

\footnotetext{
${ }^{9}$ As we assume genuine displacements dominate the backgrounds, we expect this estimate would not produce the true contents of the $d_{0} \leq 0.1 \mathrm{~mm}$ bins, but capture only those effects that scale approximately like exponentials which have not become negligibly small for $d_{0}>0.1 \mathrm{~mm}$. In particular, we would expect $t \bar{t}$ to have a very large population from prompt leptons.
} 

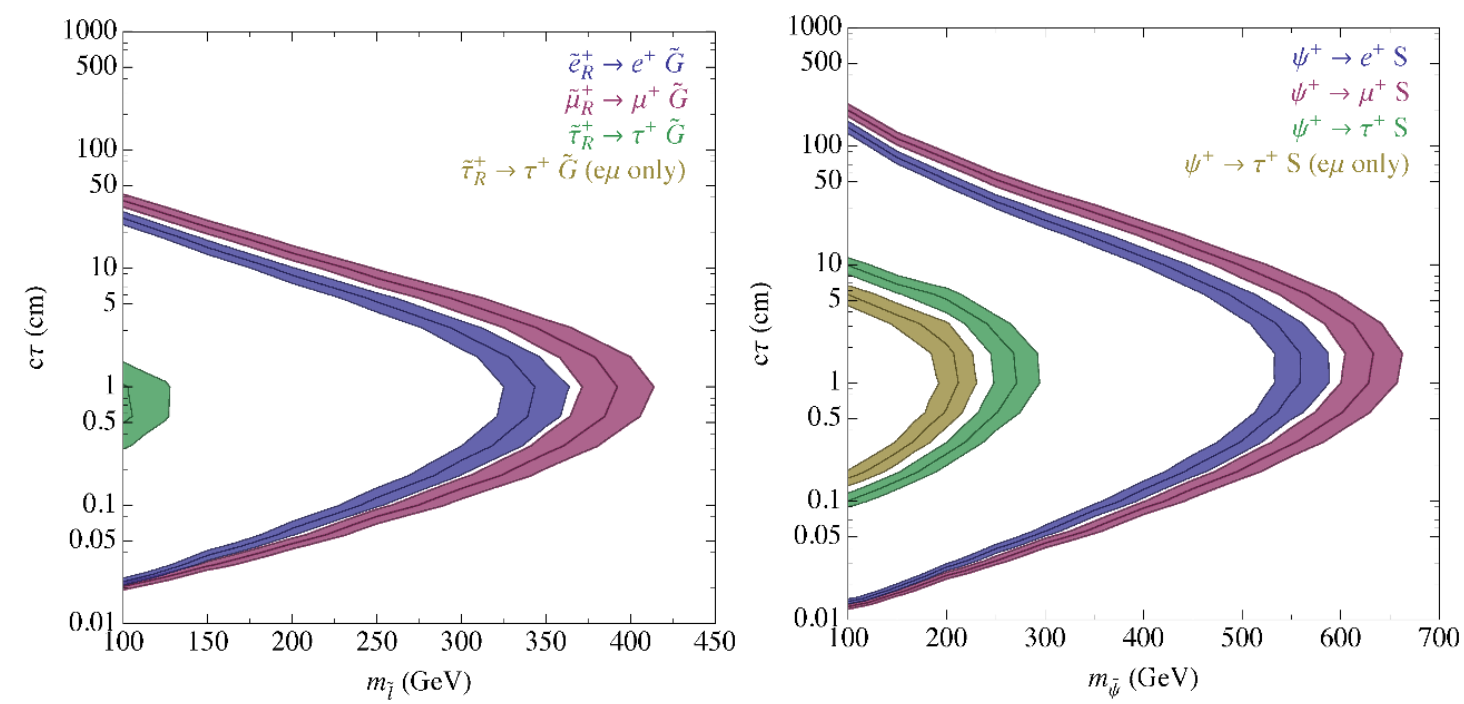

Figure 4. Left: $13 \mathrm{TeV}$ reach for direct production of a single species of slepton that decays as $\tilde{\ell} \rightarrow \ell \tilde{G}$. Using a $13 \mathrm{TeV}$ version of the CMS displaced $e \mu$ search without the same-flavor channels has no sensitivity. The width of the band reflects a $25 \%$ modeling uncertainty. Right: $13 \mathrm{TeV}$ reach for direct production of a unit charge singlet fermion that decays to a single species of lepton and a very light dark matter particle. In gold, we present limits on $\tau$-flavored dark matter without including the same-flavor channels (which are weaker by almost $100 \mathrm{GeV}$ ). We show a $25 \%$ modeling uncertainty.

amined the probability to find an isolated, displaced lepton of sufficient $p_{T}$ from a heavy flavor decay. This probability was found to be approximately independent of the boost of the parent $B$ meson. Although this information was determined from Monte Carlo and thus should be viewed with some caution, we took this as sufficient evidence that, for the purposes of this study, we could neglect the effects of altered $b \bar{b}$ kinematics and rescale the heavy flavor background by the cross-section alone. In doing this rescaling for each background, we are implicitly assuming that the tails of the distributions also scale simply with the cross-section; however, as the background estimates are rather small in SR2 and SR3 where these tails are most relevant, only an egregious underestimate would result in a qualitative change to our projected limits.

Rescaling the individual distributions from $8 \mathrm{TeV}$ to $13 \mathrm{TeV}$ and projecting the same $\mathrm{HF}$ ( $Z$ and top) systematic uncertainty of $30 \%(10 \%)$ present in the $8 \mathrm{TeV}$ data, we derive estimates for the different signal regions (table 3). Using these background projections, we can estimate the $13 \mathrm{TeV}$ sensitivity to models of direct slepton production with EGMSBlike decay chains $\tilde{e}^{ \pm} \rightarrow e \tilde{G}$ and $\tilde{\mu}^{ \pm} \rightarrow \mu \tilde{G}$. In addition to combining all nine $13 \mathrm{TeV}$ search regions (table 3 ) to project limits on a $\tilde{\tau}_{R}$ NLSP, we also show the limits from the $e \mu$ channel alone to illustrate the improvement a combination gives to the reach. Lastly, we show the reach for a lepton-flavored dark matter motivated model with an $S U(2)_{L}$ singlet charged fermion that decays as $\psi^{ \pm} \rightarrow e / \mu / \tau S$ to a light scalar dark matter $S$. This model, which has been discussed elsewhere [92] with larger $\psi \ell S$ couplings, was constructed in FeynRules [93] with all limits presented using leading-order cross-sections. All results are shown in figure 4. 
While we chose to mirror the $8 \mathrm{TeV}$ search in order to get a more reliable modeling of the background, we note that all of the same-flavor models typically predict very hard leptons (unlike in the $\tilde{\tau}$ cases). Considering not only lower $p_{T}$ thresholds essential for sensitivity to staus, but also a higher lepton $p_{T}$ threshold signal region, e.g., SR1' with $p_{T, \ell}>50 \mathrm{GeV}$, could vastly reduce backgrounds in SR1 while having minimal impact on the benchmark signal models. This additional search region could greatly increase sensitivity at lower $c \tau$ values.

\section{Discussion and conclusions}

Probing all feasible lifetimes for NLSP particles in GMSB is paramount in the search for new physics at the LHC. The very generic GMSB scenario containing an NLSP $\tilde{\tau}_{R}$ is currently under-constrained for many macroscopic stau lifetimes. The only existing search able to target the displaced leptons from $\tilde{\tau}_{R}$ decays is the CMS search for $e^{ \pm} \mu^{\mp}$ with large impact parameters [17], while HSCP [40, 41] and disappearing track searches [43, 44] can target the long-lived sleptons themselves. With the exception of the HSCP searches, the experimental analyses did not consider NLSP staus as one of their benchmark signal models, so the cuts were not tailored to probe the specific signatures of long-lived $\tilde{\tau}$ s. Only the HSCP search currently places limits beyond those of LEP on the direct production of a $\tilde{\tau}_{R}$ NLSP.

In section 2, we recast an HSCP search, two disappearing track searches, and the CMS displaced $e \mu$ search to place constraints on direct stau production, as well as on simplified models with displaced staus originating from cascade decays initiated by Higgsinos, stops, and gluinos. While we find meaningful constraints on these models, several modifications to the searches were discussed in detail that could improve sensitivity to long-lived staus. Our most important suggestion for the disappearing track searches and the CMS displaced $e \mu$ search is simply to include NLSP staus as a benchmark model. We found the recasting recommendations provided in the CMS searches to be invaluable for our recasting efforts. If the ATLAS disappearing track search were to provide efficiency maps or similar resources, it would greatly improve the reliability of any recast of their results. It may be possible to improve sensitivity to staus if the ATLAS disappearing track search were to check for energy deposits that originate from the terminus of the disappearing track and/or arrive later than typical by employing their calorimeter's exceptional pointing and timing capabilities. Similarly, CMS could use their calorimeter's timing information to permit delayed energy deposits to live within their strict isolation cone. More generally for the disappearing track searches at both experiments, an extension or related analysis that attempts to reconstruct a kinked track signature could greatly improve sensitivity to sleptons.

For the CMS displaced $e \mu$ search, which uses the most recently designed experimental strategy, we discussed in sections 2.3 and 4 several avenues to improve sensitivity to displaced $\tilde{\tau}_{R}$ NLSPs and similar signatures. We briefly summarize these proposed improvements and suggest a few other possibilities that could enhance the sensitivity:

- Leptons from boosted right-handed $\tau$ decays are typically soft, and thus lowering the $p_{T}$ thresholds as much as possible for one or both species of leptons can greatly improve signal acceptance. 
- The stringent isolation requirements could be relaxed in the higher displacement (and thus lower background) signal regions in order to increase the signal acceptance. Again, as leptons from right-handed $\tau$ decays are typically soft, relaxing the isolation criteria can have a notable impact on signal acceptance.

- The flavor-universal decay of the $\tilde{\tau}$ results in not only $e^{ \pm} \mu^{\mp}$ final states, but also $e^{+} e^{-}$ and $\mu^{+} \mu^{-}$. A combination of all channels can improve reach, especially because of the lower expected backgrounds in the case of $\mu^{+} \mu^{-}$.

- The veto on additional leptons seems unnecessary and can reduce acceptance in noisier production channels, e.g., gluino-initiated decay chains.

- The presence of Majorana particles such as gluinos or neutral Higgsinos in the decay chain can give rise to same-sign lepton signatures (as can mesino oscillation). Not only can the inclusion of same-sign lepton bins extend the reach, but the effects of the same-sign lepton signals should be considered in the context of control region contamination.

- There are 1.5 orders of magnitude in $c \tau$ between the peaks in sensitivity for the disappearing track searches and the CMS displaced $e \mu$ search, with a noticeable deficiency in the range $c \tau=3-5 \mathrm{~cm}$. For this reason, it is very important to be able to extend the search regions beyond the $d_{0}<2 \mathrm{~cm}$ range. If electron reconstruction cannot be extended to higher impact parameters, extending the range of muon reconstruction alone could still notably increase sensitivity to longer lifetimes $(c \tau \sim 10 \mathrm{~cm})$, especially as these high displacement regions are likely to remain low in background (although cosmic muon backgrounds may become more important).

- Including highly displaced hadronic taus in $e \tau_{h}, \mu \tau_{h}$, and even $\tau_{h} \tau_{h}$ channels, would improve the reach. Determining the feasibility of such a search is beyond the scope of this work, but we note this possibility as one of the most robust, if challenging, ways to extend sensitivity to long-lived $\tilde{\tau}_{R} \mathrm{~s}$.

While long-lived $\tilde{\tau}_{R} \mathrm{~s}$ are a particularly well-motivated signal model, it is worth noting on more general grounds that the current LHC search program has a gap in coverage for same-flavor solitary leptons with large impact parameters. While HSCP searches and to a lesser extent displaced track searches provide good coverage at longer lifetimes, at shorter lifetimes these signatures can be efficiently hidden from standard prompt BSM searches, thanks to the tight lepton quality criteria and cosmic muon vetoes employed by these analyses. Of the large and increasing number of LHC searches for displaced objects, only the CMS displaced $e \mu$ search is in principle sensitive to solitary displaced leptons, and would miss any model that preferentially yields same-flavor leptons. We have discussed several classes of theories which can give rise to displaced same-flavor lepton signatures, such as extended GMSB, RPV SUSY, and lepton-flavored dark matter, and have proposed specific extensions to existing search strategies to enhance discovery prospects for these signatures. Additionally, the same-flavor signature would be the best handle on models of 
GMSB with long-lived co-NLSP sleptons, and would provide valuable additional sensitivity to stau NLSPs alone. As displaced leptons are both a well-motivated exotic detector object and one of the least constrained by current searches, closing this gap is a key step in maximizing the physics potential of the LHC as Run II goes forward.

\section{Acknowledgments}

We thank F. Bishara, J. P. Chou, M. Diamond, C. Frugiuele, A. Haas, C. Hill, Y. Kats, S. Knapen, A. Monteux, and D. Shih for useful conversations. We thank L. Quertenmont and W. Wulsin for answering questions about specific searches. We thank D. Lamprea for assistance with Resummino. We are especially grateful to J. Antonelli for useful discussions and comments on the draft.

\section{A Validation of recasting procedures}

In figure 5, we present our validation results for each of the four searches we consider in detail $[17,43,44,48]$. In the case of the CMS displaced $e \mu$ search, the benchmark signal model is stop pair production with displaced $R$-parity-violating decays $\tilde{t} \rightarrow \ell_{i} b$, with equal branching fractions to each of the three species of leptons. The other three searches consider an AMSB wino model. Both the CMS HSCP and CMS disappearing track (DT) searches agree excellently across the entire parameter space. In the case of the ATLAS disappearing track search, agreement is very good for most of the parameter space, but we observe $\mathcal{O}(50 \%)$ discrepancies between our recast result and the experimental result at higher values of $c \tau$. The CMS displaced $e \mu$ search agrees very well in the region where it is most sensitive, $300 \mu \mathrm{m} \lesssim c \tau \lesssim 50 \mathrm{~cm}$, but exhibits significant deviations on the tails of sensitivity. It is likely the case that we are slightly underestimating sensitivity for lifetimes near $1 \mathrm{~m}$ or $100 \mu \mathrm{m}$, but this discrepancy has no qualitative impact on the results.

For the CMS HSCP search and the CMS displaced $e \mu$ search, we apply the recommended $25 \%$ modeling uncertainty. For both disappearing track searches we apply a $50 \%$ modeling uncertainty, primarily because of the additional uncertainty introduced by the decay products originating from the displaced secondary stau vertex.

In section 2.4, for lucidity in presentation we display at a given $(m, c \tau)$ only the stronger of the two limits from the ATLAS and CMS disappearing track searches. In figure 6, we show the sensitivity of the two disappearing track searches separately to several of the simplified signal models considered in section 2.4. In all scenarios, ATLAS has a markedly reduced sensitivity at longer lifetimes. This can be understood easily as ATLAS vetoes tracks that reach the muon chamber whereas CMS does not. Due to the presence of additional prompt leptons in the Higgsino-initiated simplified model, ATLAS vetoes more events and finds weaker limits compared to CMS. At shorter lifetimes, the ATLAS search typically performs slightly better than the CMS search, but the two set nearly identical limits after our modeling uncertainties are taken into account. 

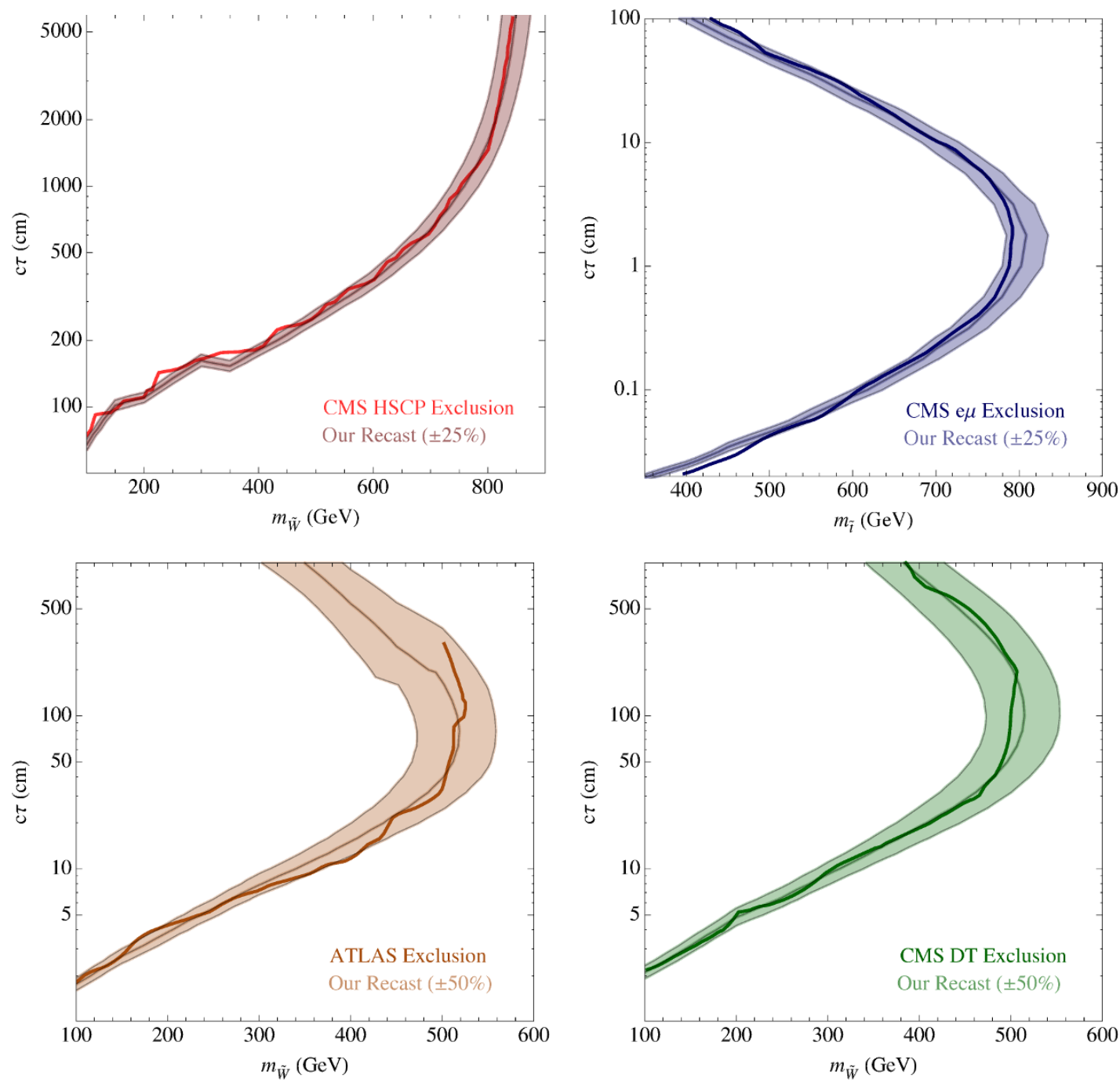

Figure 5. Upper Left: validation of the CMS search for heavy stable charged particles [48]. The width of our recast exclusion band reflects a $25 \%$ modeling uncertainty. Upper Right: validation of the CMS displaced $e \mu$ search [17] for the displaced supersymmetry benchmark model [94]. Lower Left: validation of the ATLAS disappearing tracks search [43]. Lower Right: validation of the CMS disappearing tracks search [44].

\section{B Details of the Extended Gauge Mediation Model}

In this appendix, we present a more detailed discussion of the EGMSB model presented in section 3.2. In gauge mediation, a SM singlet superfield $X$ acquires a VEV and an $F$-term, i.e., $\langle X\rangle=M+\theta^{2} F$, thereby breaking SUSY (for a nice review, see [7]). In minimal GMSB, $N$ vector-like messenger superfields $\Phi_{i}, \bar{\Phi}_{i}$ have a superpotential coupling to $X$, $W=X \Phi_{i} \bar{\Phi}_{i}$, which gives mass $M$ to the messengers. The messengers are charged under the SM gauge group and communicate SUSY breaking to the MSSM fields via gauge loops. All MSSM gauginos get soft masses at one loop,

$$
M_{\lambda_{r}}(M)=g_{r}^{2} N_{\text {eff }} \tilde{\Lambda}
$$



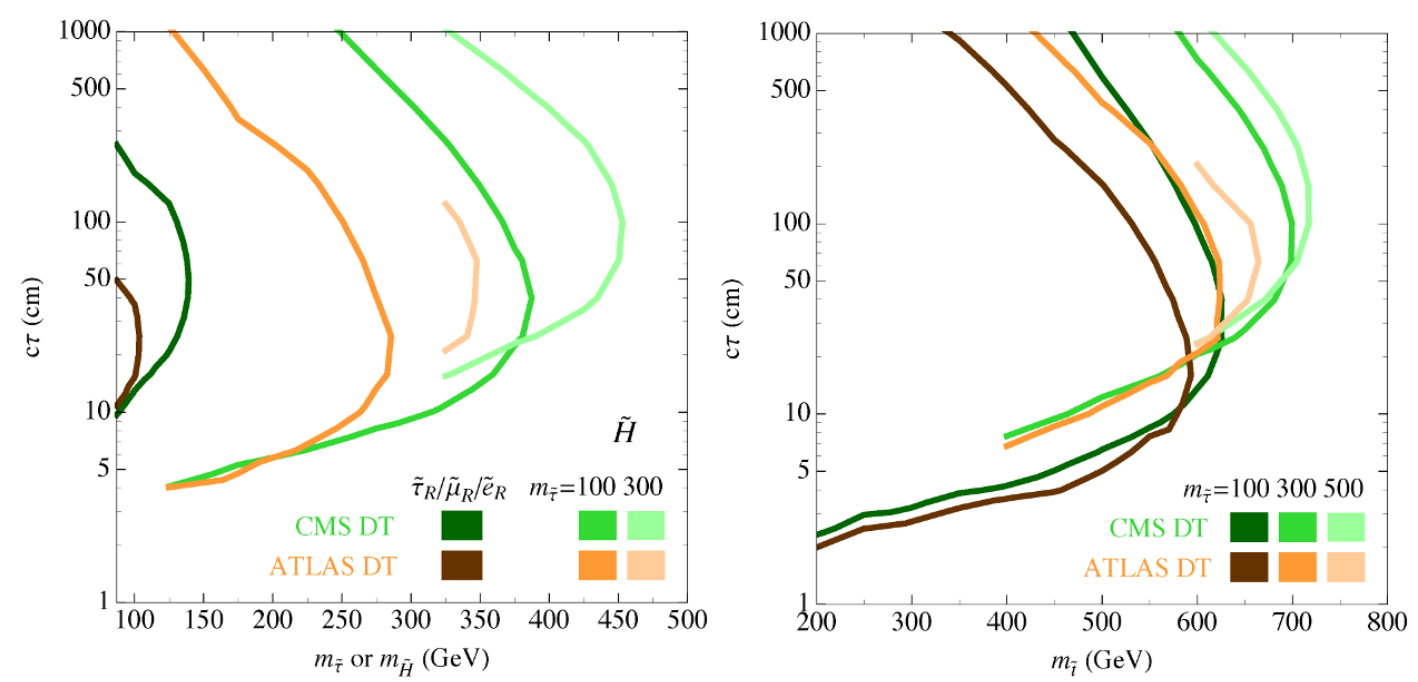

Figure 6. Left: comparison of the disappearing track searches at ATLAS [43] (orange) and CMS [44] (green) for the case of direct production of three flavors of sleptons (dark) and Higgsinos (see figure 1). Right: comparison of the disappearing track searches for the case of stops (see figure 2 right).

and all scalars get contributions to their soft masses-squared at two loops,

$$
\tilde{m}_{A}^{2}(M)=2 N_{\text {eff }} \sum_{r} c_{r} g_{r}^{4} \tilde{\Lambda}^{2} .
$$

Here we have defined $\Lambda \equiv \frac{F}{M}, \tilde{\Lambda} \equiv \frac{1}{16 \pi^{2}} \Lambda$, and the effective number of messengers $N_{\text {eff }}$ (a $5 \oplus \overline{5}$ of $\mathrm{SU}(5)$ contributes 1 and a $10 \oplus \overline{10}$ contributes 3). The quadratic Casimir of a MSSM field $c_{r}$ under the SM gauge group $r$ is, for hypercharge, normalized according to its embedding in $\mathrm{SU}(5)$ or larger grand unified theory, $c_{1}=\frac{3}{5} Y^{2}$.

In EGMSB, one introduces additional direct couplings in the superpotential between the MSSM and messenger superfields. To directly affect right-handed sleptons, these couplings should take the form

$$
W \supset \kappa_{i} E_{i}^{c} \Phi \tilde{\Phi} \quad \text { with }(\Phi, \tilde{\Phi})=\left(\Phi_{\bar{E}}, \Phi_{S}\right),\left(\Phi_{L_{1}}, \Phi_{L_{2}}\right) \text { or }\left(\Phi_{U}, \Phi_{\bar{D}}\right)
$$

where $\Phi_{A}$ is a messenger with the same gauge quantum numbers as the superfield $A$. That is, in the notation $\left(\mathrm{SU}(3)_{C}, \mathrm{SU}(2)_{L}\right)_{\mathrm{U}(1)_{Y}}$, we have: $\bar{E}=(1,1)_{-1}, L_{i}=(1,2)_{-\frac{1}{2}}$, $U=(\overline{3}, 1)_{-\frac{2}{3}}, \bar{D}=(3,1)_{-\frac{1}{3}}$, and $S=(1,1)_{0}$. Two distinct $L$ fields are required lest the coupling be identically zero. With the general formulas from [24], the EGMSB contribution to the slepton mass-squared can be written

$$
\delta \tilde{m}_{E, i j}^{2}=\left[\left(d_{E}+2\right) \kappa_{k}^{*} \kappa_{k}-2 C_{r}^{E \Phi \tilde{\Phi}} g_{r}^{2}-\frac{16 \pi^{2}}{3} h\left(\frac{\Lambda}{M}\right)\left(\frac{\Lambda}{M}\right)^{2}\right] d_{E} \kappa_{i}^{*} \kappa_{j} \tilde{\Lambda}^{2},
$$

where $d_{E}$ sums the number of messenger fields with direct superpotential couplings to $E$, the coefficient $C_{r}^{E \Phi \tilde{\Phi}}$ is the sum of the Casimirs of the operators in the superpotential coupling, $C_{r}^{E \Phi \tilde{\Phi}}=c_{r}^{E}+c_{r}^{\Phi}+c_{r}^{\tilde{\Phi}}$, and the function $h(x)$ is given by $h(x)=1+\frac{4}{5} x^{2}+$ 


\begin{tabular}{|l|cc|c|c|}
\hline Operator & $N_{\text {eff }}=|\Delta b|$ & $N_{\max }$ & $d_{E}$ & $C^{E \Phi \tilde{\Phi}}$ \\
\hline$E_{i}^{c} \Phi_{\bar{E}} \Phi_{S}$ & $3 N$ & 2 & $N$ & $\left(\frac{6}{5}, 0,0\right)$ \\
$E_{i}^{c} \Phi_{L_{1}} \Phi_{L_{2}}$ & $2 N$ & 3 & $2 N$ & $\left(\frac{9}{10}, \frac{3}{2}, 0\right)$ \\
$E_{i}^{c} \Phi_{U} \Phi_{\bar{D}}$ & $4 N$ & 1 & $3 N$ & $\left(\frac{14}{15}, 0, \frac{8}{3}\right)$ \\
\hline
\end{tabular}

Table 4. Model parameters influenced by the choice of EGMSB operator. $|\Delta b|$ is the contribution of the messengers to the $\mathrm{SU}(5)$ beta function. $N_{\max }$ is the maximum number of messengers consistent with perturbative unification, i.e., $|\Delta b| \leq 6 . C^{E \Phi \tilde{\Phi}}$ is the sum of the quadratic Casimirs of the three fields within the operator $E \Phi \tilde{\Phi}$ for the groups (U(1), SU(2), $\mathrm{SU}(3))$ respectively.

$\mathcal{O}\left(x^{4}\right)$ [21]. Contributions to all other soft masses and the trilinear $A$-terms are suppressed by $y_{\tau}$. For small values of $\kappa_{i} \ll g_{r}$, the first term in (B.4) can be neglected.

From the above expressions we can get an idea for the parameter scales involved over the masses and lifetimes of interest. For simplicity of discussion, we will neglect the effects of running. The slepton NLSP lifetime (1.1) can be inverted to give an expression for the SUSY-breaking scale in terms of the slepton mass and lifetime,

$$
F=(100 \mathrm{TeV})^{2}\left(\frac{c \tau}{100 \mu m}\right)^{\frac{1}{2}}\left(\frac{m_{\tilde{\ell}}}{100 \mathrm{GeV}}\right)^{\frac{5}{2}}
$$

and the GMSB contribution to the slepton mass (B.2) can be inverted to give $\Lambda=$ $16 \pi^{2} m_{\tilde{\ell}}\left(1 / g_{1}^{2}\right) \sqrt{5 / 6 N_{\text {eff }}}$, or

$$
\frac{\Lambda}{M}=\frac{\Lambda^{2}}{F} \sim \frac{1}{3 N_{\mathrm{eff}}}\left(\frac{100 \mu m}{c \tau}\right)^{\frac{1}{2}}\left(\frac{100 \mathrm{GeV}}{m_{\tilde{\ell}}}\right)^{\frac{1}{2}},
$$

where in the last equation we have approximated $g_{1}(M) \sim 0.5$. Now, focusing on the $\kappa_{i} E_{i}^{c} \Phi_{U} \Phi_{\bar{D}}$ model with $N=1$ for simplicity, we can use (B.4) and table 4 to see that the EGMSB contribution to the slepton mass-squared is

$$
\delta m_{\tilde{\ell}, i j}^{2}=-\left[\frac{28}{5} g_{1}^{2}+16 g_{3}^{2}+16 \pi^{2} h\left(\frac{\Lambda}{M}\right)\left(\frac{\Lambda}{M}\right)^{2}\right] \kappa_{i}^{*} \kappa_{j} \tilde{\Lambda}^{2} .
$$

Then, substituting (B.5), (B.6), and using $g_{3}(M) \sim 1$, we can approximate this expression as

$$
\delta m_{\tilde{\ell}, i j}^{2} \sim-50\left[1+\frac{1}{16}\left(\frac{100 \mu m}{c \tau}\right)\left(\frac{100 \mathrm{GeV}}{m_{\tilde{\ell}}}\right)\right] \kappa_{i}^{*} \kappa_{j} m_{\tilde{\ell}}^{2}
$$

Taking for definiteness the $\vec{\kappa}$ direction in flavor space to be aligned with the muon, we can approximate the small splitting in slepton mass (as opposed to mass squared) between $\tilde{\mu}$ and the other sleptons as

$$
\Delta m_{\tilde{\mu}} \sim 25 \kappa_{2}^{2} m_{\tilde{\ell}}
$$

which, at the lowest masses of interest, gives $\mathcal{O}(10 \mathrm{GeV})$ splittings for $\kappa_{2} \sim 6 \times 10^{-2}$. 
An analogous expression can be derived for the other messenger models. At the other extreme using the same basic approximations, the $\kappa_{i} E_{i}^{c} \Phi_{\bar{E}} \Phi_{S}$ model gives

$$
\Delta m_{\tilde{\mu}} \sim \frac{4}{3}\left[1+\frac{1}{N^{2}}\left(\frac{100 \mu m}{c \tau}\right)\left(\frac{100 \mathrm{GeV}}{m_{\tilde{\ell}}}\right)\right] \kappa_{2}^{2} m_{\tilde{\ell}}
$$

which is more sensitive to the lifetime, but typically requires $\kappa_{2} \sim 0.2-0.3$ for an $\mathcal{O}(10 \mathrm{GeV})$ splitting.

\section{Freezein during an early matter-dominated era}

In this appendix, we provide a brief discussion of dark matter freezein during an early period of matter domination. Such epochs of matter domination are created by the coherent oscillations of a heavy modulus or inflaton, $\phi$, with a late decay into relativistic species. Expansion during this time is non-adiabatic due to the entropy injection from the decay of the heavy species. This period of matter domination lasts until a time, $t \sim \Gamma_{\phi}^{-1}$, at which point enough of the heavy species have decayed so that the universe enters a radiationdominated era. The lifetime of $\phi$ determines the reheat temperature (see e.g. [95] for a review),

$$
T_{\mathrm{RH}}=\left(\frac{90}{8 \pi^{3} g_{*}}\right)^{1 / 4} \sqrt{\Gamma_{\phi} M_{\mathrm{pl}}},
$$

which is the temperature where the universe transitions into a standard radiationdominated adiabatic expansion. Importantly, at times earlier than $T_{\mathrm{RH}}$, the temperature of the thermal bath is higher than $T_{\mathrm{RH}}[85] .^{10}$

For simplicity, we will consider DM freezein during the period of reheating following inflation, and take $\phi$ to be the inflaton; periods of modulus-domination yield quantitatively similar results in our region of interest. The Boltzmann equations describing the evolution of the energy density stored in the inflaton field, $\rho_{\phi}$, and the energy density stored in the relativistic species, $\rho_{R}$, are

$$
\begin{gathered}
\dot{\rho}_{\phi}+3 H \rho_{\phi}=-\Gamma_{\phi} \rho_{\phi} \\
\dot{\rho}_{R}+4 H \rho_{R}=\Gamma_{\phi} \rho_{\phi} .
\end{gathered}
$$

Here, $a$ is the scale factor, and $H=\dot{a} / a$ is the Hubble parameter. We define the co-moving quantities

$$
\Phi \equiv \rho_{\phi} a^{3} \quad \text { and } \quad R \equiv \rho_{R} a^{4} .
$$

Using the Friedmann equation

$$
H^{2}=\frac{8 \pi}{3} \frac{1}{M_{\mathrm{pl}}^{2} a^{4}}(a \Phi+R)
$$

\footnotetext{
${ }^{10}$ We assume that the decay products of the heavy scalar field have reached thermal equilibrium at some temperature above the scale where freezein becomes relevant.
} 
the Boltzmann equations (C.2- C.3) can be rewritten as

$$
\begin{aligned}
\Phi^{\prime} & =-\mathcal{C} \frac{a}{\sqrt{a \Phi+R}} \Phi \\
R^{\prime} & =\mathcal{C} \frac{a^{2}}{\sqrt{a \Phi+R}} \Phi
\end{aligned}
$$

where we have defined

$$
\mathcal{C} \equiv \sqrt{\frac{3}{8 \pi}} M_{\mathrm{pl}} \Gamma_{\phi}
$$

Initial conditions are determined from the end of inflation, which occurs at some scale $a_{i}$, with inflaton energy density $\rho_{\phi}\left(a_{i}\right) \equiv \rho_{\phi, i}$, while $\rho_{R}\left(a_{i}\right)=0$. In practice, the late-time behavior of the system is insensitive to the exact values of $a_{i}$ and $\rho_{\phi, i}$.

In general, these equations must be solved numerically. However, it is useful to construct an approximate analytic solution to this system as follows. First, we approximate the transition from matter-domination to radiation-domination as an instantaneous energy transfer at $a_{\mathrm{RH}}$, so that $\rho_{\phi}\left(a_{\mathrm{RH}}^{-}\right)=\rho_{R}\left(a_{\mathrm{RH}}^{+}\right)$. A second simplifying assumption is to neglect the effect of $\Gamma_{\phi}$ on $\Phi$ until this instantaneous transfer. Thirdly, we take the Hubble parameter to depend only on $\Phi$ prior to $T_{\mathrm{RH}}$, neglecting the small contribution of the radiation. As long as we are not concerned with the detailed behavior near the transition from matter-domination to radiation-domination, i.e., near $T_{\mathrm{RH}}$, these are excellent approximations. With these approximations, eqs. (C.6 - C.7) can be simply integrated to yield, at leading order,

$$
R(a) \approx \frac{2 \mathcal{C}}{5} \Phi_{i}^{1 / 2}\left(a^{5 / 2}-a_{i}^{5 / 2}\right) \approx \frac{2 \mathcal{C}}{5} \Phi_{\mathrm{RH}}^{1 / 2}\left(a^{5 / 2}-a_{i}^{5 / 2}\right),
$$

where our approximations allow us to further express $\Phi_{\mathrm{RH}}$ simply in terms of the reheating temperature and $a_{\mathrm{RH}}$. From this expression, it is evident that for $a \gg a_{i}$, dependence on the detailed choice of $a_{i}$ drops out.

The radiation energy density defines an expression for temperature. In the matterdominated regime, we have

$$
T(a)=\frac{1}{a}\left(\frac{30 \mathcal{C}}{\pi^{2} g_{*}(T)}\right)^{1 / 4}\left[\frac{2}{5} \Phi_{i}^{1 / 2}\left(a^{5 / 2}-a_{i}^{5 / 2}\right)\right]^{1 / 4} .
$$

From this relation, it is easy to see that the temperature scales approximately as

$$
T \propto a^{-3 / 8} .
$$

We are interested in the freezein production of DM during the matter-dominated epoch prior to $T_{\mathrm{RH}}$. The Boltzmann equation governing the freezein of scalar lepton-flavored dark matter through the decay of a fermionic charged parent, $\psi$, in thermal equilibrium can be written as

$$
\dot{n}_{S}+3 H n_{S}=\int d \Pi_{\psi} d \Pi_{S} d \Pi_{\ell}(2 \pi)^{4} \delta^{4}\left(\sum p_{i}\right)|\overline{\mathcal{M}}(\psi \rightarrow S \ell)|^{2} f_{\psi}\left(1-f_{\ell}\right)\left(1+f_{S}\right),
$$


where we have taken all other dark matter interaction rates, including the inverse process $\ell S \rightarrow \psi$, to be negligible. To simplify (C.12), we note that $f_{S} \ll 1$ and approximate $\left(1-f_{\ell}\right) \approx 1$, giving [82]

$$
\dot{n}_{S}+3 H n_{S} \approx \int d \Pi_{\psi} 2 m_{\psi} \Gamma_{\psi} f_{\psi}=\frac{g_{\psi} \Gamma_{\psi} m_{\psi}}{2 \pi^{2}} \int_{m_{\psi}}^{\infty} \sqrt{E^{2}-m_{\psi}^{2}} f_{\psi} d E
$$

where $g_{\psi}=4$ is the number of internal degrees of freedom for $\psi$. Since temperatures where $T \sim m_{\psi} /\{$ few $\}$ dominate freezein, using a Maxwell-Boltzmann approximation for $f_{\psi}$ is not an unreasonable approximation, but will yields a slightly higher dark matter density than that obtained by using Fermi-Dirac statistics. Defining the quantity $\mathcal{S} \equiv n_{S} a^{3} / \Gamma_{\psi}$, we can simplify (C.13) to

$$
\Gamma_{\psi} \mathcal{S}^{\prime}=\frac{a^{2}}{H} \frac{g_{\psi} \Gamma_{\psi} m_{\psi}^{2} T}{2 \pi^{2}} K_{1}\left(\frac{m_{\psi}}{T}\right) I_{\mathrm{FD}}\left(\frac{m_{\psi}}{T}\right),
$$

where $K_{1}(x)$ is a modified Bessel function of the second kind, and the monotonic function

$$
I_{\mathrm{FD}}(x)=\frac{1}{x K_{1}(x)}\left(\int_{x}^{\infty} \frac{\sqrt{u^{2}-x^{2}}}{1+e^{u}} d u\right) \approx \begin{cases}\frac{\pi^{2}}{12} \approx 0.822 & x \ll 1 \\ 1 & x \gg 1\end{cases}
$$

encapsulates the departure from the Maxwell-Boltzmann approximation.

Using (C.5) and (C.10), equation (C.14) can be numerically integrated as

$$
\mathcal{S}\left(a^{\prime}\right)=\frac{g_{\psi} m_{\psi}^{2}}{2 \pi^{2}} \int_{a_{i}}^{a^{\prime}} \frac{a^{2} T(a)}{H(a)} K_{1}\left(\frac{m_{\psi}}{T(a)}\right) I_{\mathrm{FD}}\left(\frac{m_{\psi}}{T(a)}\right) d a .
$$

As long as $T_{\mathrm{RH}}$ is sufficiently smaller than $m_{\psi}$, our simplifying assumptions about the transition from matter-domination to radiation-domination are reliable approximations. After $a_{\mathrm{RH}}$, the universe expands adiabatically, so $\mathcal{S}$ is constant during this era. We can then relate this quantity to the present day dark matter abundance,

$$
\Omega_{\mathrm{DM}}=\frac{m_{S} n_{S, 0}}{\rho_{\text {crit }, 0}}=m_{S} \Gamma_{\psi} \frac{\mathcal{S}\left(a_{0}\right)}{a_{0}^{3} \rho_{\text {crit }, 0}}=m_{S} \Gamma_{\psi} \frac{\mathcal{S}\left(a_{\mathrm{RH}}\right)}{a_{0}^{3} \rho_{\text {crit }, 0}}=m_{S} \Gamma_{\psi} \frac{\mathcal{S}\left(T_{\mathrm{RH}}, m_{\psi}, \frac{a_{\mathrm{RH}}}{a_{i}}\right)}{\rho_{\text {crit }, 0}},
$$

where $a_{0} \equiv 1$ is the scale factor today and $\rho_{\text {crit }, 0}=3.80 \times 10^{-47} \mathrm{GeV}^{4}$ is the critical energy density of the universe. In the last equality, $\mathcal{S}\left(a_{\mathrm{RH}}\right)$ has been written explicitly in terms of all parameters on which it depends. As long as the matter-dominated era is sufficiently long, i.e., $a_{\mathrm{RH}} / a_{i} \gg\left(m_{\psi} / T_{\mathrm{RH}}\right)^{8 / 3}, \mathcal{S}$ is insensitive to $a_{i}$. Assuming this condition on $a_{i}$ holds, we can write (C.17) as

$$
\frac{\Omega_{\mathrm{DM}}}{\Omega_{\mathrm{DM}, \mathrm{obs}}}=\left(\frac{\mathcal{S}\left(T_{\mathrm{RH}}, m_{\psi}\right)}{5.2 \times 10^{-31} \mathrm{GeV}^{2}}\right)\left(\frac{m_{S}}{1 \mathrm{MeV}}\right)\left(\frac{1 \mathrm{~cm}}{c \tau_{\psi}}\right) .
$$

The scaling relations derived in ref. [87] illustrate that $\mathcal{S}\left(T_{\mathrm{RH}}, m_{\psi}\right) \propto T_{\mathrm{RH}}^{7} / m_{\psi}^{9}$. Noting this relation, we can extract the correct numerical factors to express (C.18) approximately as

$$
\frac{\Omega_{\mathrm{DM}}}{\Omega_{\mathrm{DM}, \mathrm{obs}}} \approx\left(\frac{20}{m_{\psi} / T_{\mathrm{RH}}}\right)^{7}\left(\frac{500 \mathrm{GeV}}{m_{\psi}}\right)^{2}\left(\frac{m_{S}}{1 \mathrm{MeV}}\right)\left(\frac{1 \mathrm{~cm}}{c \tau_{\psi}}\right) .
$$


Equation (C.19) can fix one of the remaining four parameters: $c \tau_{\psi}, m_{\psi}, m_{S}$, or $T_{\mathrm{RH}}$. Thus, displaced decays at the LHC imply a relatively low reheat temperature, $T_{\mathrm{RH}} \lesssim \mathrm{TeV}$.

From (C.19), it would appear that for specific collider parameters $c \tau_{\psi}$ and $m_{\psi}$, one can always choose $T_{\mathrm{RH}}$ and $m_{S}$ to produce the correct dark matter relic abundance. However, if at some point in the early universe the number density of dark matter becomes too large, the neglected rate for the inverse process $\ell S \rightarrow \psi$ will become important. To estimate the range of validity of the the above calculation, we will require that the DM number density satisfies

$$
n_{S}(T)<k_{\text {crit }} n_{S, e q}(T)=k_{\text {crit }} \frac{\zeta(3)}{\pi^{2}} T^{3}
$$

where $\zeta(3) \approx 1.202$ is the Riemann zeta function, $n_{S, e q}(T)$ is the equilibrium number density of a relativistic scalar particle in thermal equilibrium, and $k_{\text {crit }}<1$ is a measure of when (C.12) ceases to be reliable. As the freezein mechanism does not produce a thermal distribution for the dark matter and $f_{\psi}(p) \leq f^{e q}(p)$ [96], a numerical study beyond the scope of this work would be required to determine precisely where these rates become comparable.

As the bulk of freezein happens near $T \sim m_{\psi} /\{$ few $\}$, we are interested in (C.20) applied near $T_{F I} \approx m_{\psi} / 4$. After freezein $\left(T<T_{F I} \approx m_{\psi} / 4\right)$, production of dark matter is negligible, so the number density simply redshifts with the expanding universe,

$$
n_{S}\left(a_{F I}\right)=n_{S}\left(a_{\mathrm{RH}}\right) \frac{a_{\mathrm{RH}}^{3}}{a_{F I}^{3}}=n_{S}\left(a_{\mathrm{RH}}\right)\left(\frac{T_{F I}}{T_{\mathrm{RH}}}\right)^{8},
$$

where we have used (C.11). Of course, $n_{S}\left(a_{\mathrm{RH}}\right)$ can be directly related to the dark matter density today,

$$
n_{S}\left(a_{\mathrm{RH}}\right)=\frac{\Omega_{\mathrm{DM}}}{m_{S}} \frac{\rho_{\mathrm{crit}, 0}}{s_{0}} s_{\mathrm{RH}}=\frac{\Omega_{\mathrm{DM}}}{m_{S}} \frac{\rho_{\mathrm{crit}, 0}}{s_{0}} \frac{2 \pi^{2}}{45} g_{* S}\left(T_{\mathrm{RH}}\right) T_{\mathrm{RH}}^{3}
$$

where $s_{0}=2.22 \times 10^{-38} \mathrm{GeV}^{3}$ is the entropy density today. Combining (C.20)-(C.22), we derive

$$
k_{\text {crit }}>\frac{2 \pi^{4}}{45 \zeta(3)} \frac{\Omega_{\mathrm{DM}}}{m_{S}} \frac{\rho_{\text {crit }, 0}}{s_{0}} g_{* S}\left(T_{\mathrm{RH}}\right)\left(\frac{T_{F I}}{T_{\mathrm{RH}}}\right)^{5},
$$

as the region where (C.12) is reliable. While this condition is by necessity simplified, for much of the parametric range of interest for collider phenomenology $\left(100 \mathrm{GeV} \lesssim m_{\phi} \lesssim\right.$ $1 \mathrm{TeV} ; 100 \mu \mathrm{m} \lesssim c \tau \lesssim 1 \mathrm{~m})$, it is possible to choose $T_{\mathrm{RH}}$ so that the dark matter relic abundance is satisfied for $m_{S} \ll m_{\psi}$, while satisfying (C.23). However, at low $\psi$ masses and short $\psi$ lifetimes, requiring $m_{S} \ll m_{\psi}$ can lead to difficulty with (C.23). This will result in a net reduction of the DM relic abundance relative to (C.19), due to the additional depletion of the DM. When this is the case, $S$ will make up only a fraction of the current abundance, and some other particle(s) must constitute the rest. Of course, the dark matter could also have $m_{S} \sim m_{\psi}$, so that its mass substantially influences collider kinematics. Although these are interesting possibilities, in order to simplify our presentation, we will take $m_{S} \ll m_{\psi}$ throughout this work. 
Open Access. This article is distributed under the terms of the Creative Commons Attribution License (CC-BY 4.0), which permits any use, distribution and reproduction in any medium, provided the original author(s) and source are credited.

\section{References}

[1] E. Halkiadakis, G. Redlinger and D. Shih, Status and implications of beyond-the-standard-model searches at the LHC, Ann. Rev. Nucl. Part. Sci. 64 (2014) 319 [arXiv: 1411.1427] [INSPIRE].

[2] J.A. Evans, Flavors of supersymmetry beyond vanilla, arXiv:1509.08504 [INSPIRE].

[3] R. Barbier et al., R-parity violating supersymmetry, Phys. Rept. 420 (2005) 1 [hep-ph/0406039] [INSPIRE].

[4] J. Fan, M. Reece and J.T. Ruderman, Stealth supersymmetry, JHEP 11 (2011) 012 [arXiv: 1105.5135] [INSPIRE].

[5] J.A. Evans, Y. Kats, D. Shih and M.J. Strassler, Toward full LHC coverage of natural supersymmetry, JHEP 07 (2014) 101 [arXiv: 1310.5758] [INSPIRE].

[6] J. Fan, R. Krall, D. Pinner, M. Reece and J.T. Ruderman, Stealth supersymmetry simplified, arXiv: 1512.05781 [INSPIRE].

[7] G.F. Giudice and R. Rattazzi, Theories with gauge mediated supersymmetry breaking, Phys. Rept. 322 (1999) 419 [hep-ph/9801271] [INSPIRE].

[8] A. Arvanitaki, N. Craig, S. Dimopoulos and G. Villadoro, Mini-split, JHEP 02 (2013) 126 [arXiv: 1210.0555$]$ [INSPIRE].

[9] S.K. Gupta, B. Mukhopadhyaya and S.K. Rai, Right-chiral sneutrinos and long-lived staus: event characteristics at the large hadron collider, Phys. Rev. D 75 (2007) 075007 [hep-ph/0701063] [INSPIRE].

[10] Z. Liu and B. Tweedie, The fate of long-lived superparticles with hadronic decays after LHC Run 1, JHEP 06 (2015) 042 [arXiv: 1503.05923] [INSPIRE].

[11] C. Csáki, E. Kuflik, S. Lombardo, O. Slone and T. Volansky, Phenomenology of a long-lived LSP with R-parity violation, JHEP 08 (2015) 016 [arXiv: 1505.00784] [INSPIRE].

[12] N. Zwane, Long-lived particle searches in R-parity violating MSSM, arXiv:1505.03479 [INSPIRE].

[13] ATLAS collaboration, Search for long-lived neutral particles decaying into lepton jets in proton-proton collisions at $\sqrt{s}=8 \mathrm{TeV}$ with the ATLAS detector, JHEP 11 (2014) 088 [arXiv: 1409.0746] [INSPIRE].

[14] CMS collaboration, Search for long-lived particles that decay into final states containing two electrons or two muons in proton-proton collisions at $\sqrt{s}=8$ TeV, Phys. Rev. D 91 (2015) 052012 [arXiv: 1411.6977] [INSPIRE].

[15] ATLAS collaboration, Search for massive, long-lived particles using multitrack displaced vertices or displaced lepton pairs in pp collisions at $\sqrt{s}=8 \mathrm{TeV}$ with the ATLAS detector, Phys. Rev. D 92 (2015) 072004 [arXiv: 1504.05162] [INSPIRE].

[16] ATLAS collaboration, Search for long-lived, heavy particles in final states with a muon and multi-track displaced vertex in proton-proton collisions at $\sqrt{s}=7$ TeV with the ATLAS detector, Phys. Lett. B 719 (2013) 280 [arXiv:1210.7451] [INSPIRE]. 
[17] CMS collaboration, Search for displaced supersymmetry in events with an electron and a muon with large impact parameters, Phys. Rev. Lett. 114 (2015) 061801 [arXiv:1409.4789] [INSPIRE].

[18] ATLAS collaboration, Observation of a new particle in the search for the Standard Model Higgs boson with the ATLAS detector at the LHC, Phys. Lett. B 716 (2012) 1 [arXiv: 1207.7214] [INSPIRE].

[19] CMS collaboration, Observation of a new boson at a mass of $125 \mathrm{GeV}$ with the CMS experiment at the LHC, Phys. Lett. B 716 (2012) 30 [arXiv:1207.7235] [INSPIRE].

[20] P. Draper, P. Meade, M. Reece and D. Shih, Implications of a 125 GeV Higgs for the MSSM and low-scale SUSY breaking, Phys. Rev. D 85 (2012) 095007 [arXiv:1112.3068] [InSPIRE].

[21] N. Craig, S. Knapen, D. Shih and Y. Zhao, A complete model of low-scale gauge mediation, JHEP 03 (2013) 154 [arXiv: 1206.4086] [INSPIRE].

[22] M. Abdullah, I. Galon, Y. Shadmi and Y. Shirman, Flavored gauge mediation, a heavy Higgs and supersymmetric alignment, JHEP 06 (2013) 057 [arXiv: 1209.4904] [INSPIRE].

[23] P. Byakti and T.S. Ray, Burgeoning the Higgs mass to 125 GeV through messenger-matter interactions in GMSB models, JHEP 05 (2013) 055 [arXiv:1301.7605] [INSPIRE].

[24] J.A. Evans and D. Shih, Surveying extended GMSB models with $m_{h}=125 \mathrm{GeV}$, JHEP 08 (2013) 093 [arXiv: 1303.0228] [INSPIRE].

[25] L. Calibbi, P. Paradisi and R. Ziegler, Gauge mediation beyond minimal flavor violation, JHEP 06 (2013) 052 [arXiv: 1304.1453] [INSPIRE].

[26] S. Knapen and D. Shih, Higgs mediation with strong hidden sector dynamics, JHEP 08 (2014) 136 [arXiv:1311.7107] [INSPIRE].

[27] R. Ding, T. Li, F. Staub and B. Zhu, Focus point supersymmetry in extended gauge mediation, JHEP 03 (2014) 130 [arXiv:1312.5407] [INSPIRE].

[28] A. Basirnia, D. Egana-Ugrinovic, S. Knapen and D. Shih, 125 GeV Higgs from tree-level A-terms, JHEP 06 (2015) 144 [arXiv: 1501.00997] [INSPIRE].

[29] W. Fischler and W. Tangarife, Vector-like fields, messenger mixing and the Higgs mass in gauge mediation, JHEP 05 (2014) 151 [arXiv:1310.6369] [INSPIRE].

[30] C. Liu and Z.-h. Zhao, A realization of effective SUSY with strong unification, Phys. Rev. D 89 (2014) 057701 [arXiv: 1312.7389] [INSPIRE].

[31] B. Allanach, M. Badziak, C. Hugonie and R. Ziegler, Light sparticles from a light singlet in gauge mediation, Phys. Rev. D 92 (2015) 015006 [arXiv:1502.05836] [INSPIRE].

[32] A. Delgado, M. Garcia-Pepin and M. Quirós, GMSB with light stops, JHEP 08 (2015) 159 [arXiv: 1505.07469] [INSPIRE].

[33] P. Batra, A. Delgado, D.E. Kaplan and T.M.P. Tait, The Higgs mass bound in gauge extensions of the minimal supersymmetric standard model, JHEP 02 (2004) 043 [hep-ph/0309149] [INSPIRE].

[34] C. Cheung and H.L. Roberts, Higgs mass from D-terms: a Litmus test, JHEP 12 (2013) 018 [arXiv: 1207.0234] [INSPIRE].

[35] N. Craig and A. Katz, A supersymmetric Higgs sector with chiral D-terms, JHEP 05 (2013) 015 [arXiv: 1212.2635] [INSPIRE]. 
[36] X. Lu, H. Murayama, J.T. Ruderman and K. Tobioka, A natural Higgs mass in supersymmetry from non-decoupling effects, Phys. Rev. Lett. 112 (2014) 191803 [arXiv: 1308.0792] [INSPIRE].

[37] M. McGarrie, G. Moortgat-Pick and S. Porto, Confronting Higgs couplings from D-term extensions and Natural SUSY at the LHC and ILC, Eur. Phys. J. C 75 (2015) 150 [arXiv: 1411.2040] [INSPIRE].

[38] E. Bertuzzo and C. Frugiuele, Natural SM-like 126 GeV Higgs boson via nondecoupling D terms, Phys. Rev. D 93 (2016) 035019 [arXiv:1412.2765] [InSPIRE].

[39] OPAL collaboration, G. Abbiendi et al., Searches for gauge-mediated supersymmetry breaking topologies in $e^{+} e^{-}$collisions at LEP2, Eur. Phys. J. C 46 (2006) 307 [hep-ex/0507048] [INSPIRE].

[40] CMS collaboration, Searches for long-lived charged particles in pp collisions at $\sqrt{s}=7$ and 8 TeV, JHEP 07 (2013) 122 [arXiv: 1305.0491] [INSPIRE].

[41] ATLAS collaboration, Searches for heavy long-lived charged particles with the ATLAS detector in proton-proton collisions at $\sqrt{s}=8 \mathrm{TeV}$, JHEP 01 (2015) 068 [arXiv:1411.6795] [INSPIRE].

[42] J. Heisig and J. Kersten, Long-lived staus from strong production in a simplified model approach, Phys. Rev. D 86 (2012) 055020 [arXiv: 1203.1581] [INSPIRE].

[43] ATLAS collaboration, Search for charginos nearly mass degenerate with the lightest neutralino based on a disappearing-track signature in pp collisions at $\sqrt{s}=8 \mathrm{TeV}$ with the ATLAS detector, Phys. Rev. D 88 (2013) 112006 [arXiv:1310.3675] [INSPIRE].

[44] CMS collaboration, Search for disappearing tracks in proton-proton collisions at $\sqrt{s}=8$ TeV, JHEP 01 (2015) 096 [arXiv:1411.6006] [INSPIRE].

[45] S. Dimopoulos, M. Dine, S. Raby and S.D. Thomas, Experimental signatures of low-energy gauge mediated supersymmetry breaking, Phys. Rev. Lett. 76 (1996) 3494 [hep-ph/9601367] [INSPIRE].

[46] S. Ambrosanio, G.D. Kribs and S.P. Martin, Signals for gauge mediated supersymmetry breaking models at the CERN LEP-2 collider, Phys. Rev. D 56 (1997) 1761 [hep-ph/9703211] [INSPIRE].

[47] J.T. Ruderman and D. Shih, Slepton co-NLSPs at the Tevatron, JHEP 11 (2010) 046 [arXiv: 1009.1665] [INSPIRE].

[48] CMS collaboration, Constraints on the pMSSM, AMSB model and on other models from the search for long-lived charged particles in proton-proton collisions at $\sqrt{s}=8 \mathrm{TeV}$, Eur. Phys. J. C 75 (2015) 325 [arXiv:1502.02522] [INSPIRE].

[49] ATLAS collaboration, Search for pair-produced long-lived neutral particles decaying in the ATLAS hadronic calorimeter in pp collisions at $\sqrt{s}=8 \mathrm{TeV}$, Phys. Lett. B 743 (2015) 15 [arXiv: 1501.04020] [INSPIRE].

[50] ATLAS collaboration, Search for long-lived, weakly interacting particles that decay to displaced hadronic jets in proton-proton collisions at $\sqrt{s}=8$ TeV with the ATLAS detector, Phys. Rev. D 92 (2015) 012010 [arXiv:1504.03634].

[51] J. Alwall, M. Herquet, F. Maltoni, O. Mattelaer and T. Stelzer, MadGraph 5: going beyond, JHEP 06 (2011) 128 [arXiv: 1106.0522] [INSPIRE]. 
[52] K. Hagiwara, T. Li, K. Mawatari and J. Nakamura, TauDecay: a library to simulate polarized $\tau$ decays via FeynRules and MadGraph5, Eur. Phys. J. C 73 (2013) 2489 [arXiv: 1212.6247] [INSPIRE].

[53] T. Sjöstrand, S. Mrenna and P.Z. Skands, A brief introduction to PYTHIA 8.1, Comput. Phys. Commun. 178 (2008) 852 [arXiv:0710.3820] [INSPIRE].

[54] CMS collaboration, Performance of the CMS missing transverse momentum reconstruction in pp data at $\sqrt{s}=8 \mathrm{TeV}, 2015$ JINST $10 \mathrm{P} 02006$ [arXiv:1411.0511] [INSPIRE].

[55] M. Krämer et al., Supersymmetry production cross sections in pp collisions at $\sqrt{s}=7 \mathrm{TeV}$, arXiv:1206.2892 [INSPIRE].

[56] W. Beenakker, R. Hopker and M. Spira, PROSPINO: a program for the production of supersymmetric particles in next-to-leading order QCD, hep-ph/9611232 [INSPIRE].

[57] B. Fuks, M. Klasen, D.R. Lamprea and M. Rothering, Precision predictions for electroweak superpartner production at hadron colliders with Resummino, Eur. Phys. J. C 73 (2013) 2480 [arXiv: 1304.0790] [INSPIRE].

[58] CMS collaboration, Supplementary material from constraints on the pMSSM, AMSB model and on other models from the search for long-lived charged particles in proton-proton collisions at $\sqrt{s}=8 \mathrm{TeV}$, arXiv:1502.02522 (2015).

[59] L. Randall and R. Sundrum, Out of this world supersymmetry breaking, Nucl. Phys. B 557 (1999) 79 [hep-th/9810155] [INSPIRE].

[60] G.F. Giudice, M.A. Luty, H. Murayama and R. Rattazzi, Gaugino mass without singlets, JHEP 12 (1998) 027 [hep-ph/9810442] [INSPIRE].

[61] CMS Collaboration, Search for displaced SUSY in dilepton final states, CMS-PAS-B2G-12-024 (2014).

[62] CMS collaboration, Displaced SUSY parametrisation study for user, available online (2014).

[63] P.J. Fox, A.E. Nelson and N. Weiner, Dirac gaugino masses and supersoft supersymmetry breaking, JHEP 08 (2002) 035 [hep-ph/0206096] [INSPIRE].

[64] ATLAS collaboration, Search for nonpointing and delayed photons in the diphoton and missing transverse momentum final state in $8 \mathrm{TeV}$ pp collisions at the LHC using the ATLAS detector, Phys. Rev. D 90 (2014) 112005 [arXiv: 1409.5542] [INSPIRE].

[65] CMS collabroation, Search for long-lived neutral particles in the final state of delayed photons and missing energy in proton-proton collisions at $\sqrt{s}=8 \mathrm{TeV}$, CMS-PAS-EXO-12-035 (2015).

[66] Y.-S. Tsai, Decay correlations of heavy leptons in $e^{+} e^{-} \rightarrow \ell^{+} \ell^{-}$, Phys. Rev. D 4 (1971) 2821 [Erratum ibid. D 13 (1976) 771] [INSPIRE].

[67] K. Hagiwara, A.D. Martin and D. Zeppenfeld, $\tau$ polarization measurements at LEP and SLC, Phys. Lett. B 235 (1990) 198 [InSPIRE].

[68] Particle Data Group collaboration, K.A. Olive et al., Review of particle physics, Chin. Phys. C 38 (2014) 090001 [inSPIRE].

[69] U. Sarid and S.D. Thomas, Mesino-anti-mesino oscillations, Phys. Rev. Lett. 85 (2000) 1178 [hep-ph/9909349] [INSPIRE].

[70] J.A. Evans and Y. Kats, LHC coverage of RPV MSSM with light stops, JHEP 04 (2013) 028 [arXiv: 1209.0764] [INSPIRE]. 
[71] CMS collaboration, Search for long-lived neutral particles decaying to quark-antiquark pairs in proton-proton collisions at $\sqrt{s}=8$ TeV, Phys. Rev. D 91 (2015) 012007 [arXiv: 1411.6530] [INSPIRE].

[72] Z. Chacko and E. Ponton, Yukawa deflected gauge mediation, Phys. Rev. D 66 (2002) 095004 [hep-ph/0112190] [INSPIRE].

[73] Y. Shadmi and P.Z. Szabo, Flavored gauge-mediation, JHEP 06 (2012) 124 [arXiv: 1103.0292] [INSPIRE].

[74] L. Calibbi, A. Mariotti, C. Petersson and D. Redigolo, Selectron NLSP in gauge mediation, JHEP 09 (2014) 133 [arXiv: 1405.4859] [INSPIRE].

[75] J.A. Evans, D. Shih and A. Thalapillil, Chiral flavor violation from extended gauge mediation, JHEP 07 (2015) 040 [arXiv:1504.00930] [INSPIRE].

[76] MEG collaboration, J. Adam et al., New constraint on the existence of the $\mu^{+} \rightarrow e^{+} \gamma$ decay, Phys. Rev. Lett. 110 (2013) 201801 [arXiv:1303.0754] [INSPIRE].

[77] T. Jelinski and J. Pawelczyk, Masses and FCNC in flavoured GMSB scheme, arXiv: 1406.4001 [INSPIRE].

[78] L. Calibbi, P. Paradisi and R. Ziegler, Lepton flavor violation in flavored gauge mediation, Eur. Phys. J. C 74 (2014) 3211 [arXiv:1408.0754] [InSPIRE].

[79] P. Meade, N. Seiberg and D. Shih, General gauge mediation, Prog. Theor. Phys. Suppl. 177 (2009) 143 [arXiv:0801.3278] [INSPIRE].

[80] S. Kraml and D.T. Nhung, Three-body decays of sleptons in models with non-universal Higgs masses, JHEP 02 (2008) 061 [arXiv:0712.1986] [INSPIRE].

[81] P. Agrawal, S. Blanchet, Z. Chacko and C. Kilic, Flavored dark matter and its implications for direct detection and colliders, Phys. Rev. D 86 (2012) 055002 [arXiv:1109.3516] [INSPIRE].

[82] L.J. Hall, K. Jedamzik, J. March-Russell and S.M. West, Freeze-in production of FIMP dark matter, JHEP 03 (2010) 080 [arXiv:0911.1120] [INSPIRE].

[83] A. Boyarsky, J. Lesgourgues, O. Ruchayskiy and M. Viel, Lyman- $\alpha$ constraints on warm and on warm-plus-cold dark matter models, JCAP 05 (2009) 012 [arXiv:0812.0010] [INSPIRE].

[84] A. Boyarsky, O. Ruchayskiy and D. Iakubovskyi, A lower bound on the mass of dark matter particles, JCAP 03 (2009) 005 [arXiv:0808.3902] [INSPIRE].

[85] D.J.H. Chung, E.W. Kolb and A. Riotto, Production of massive particles during reheating, Phys. Rev. D 60 (1999) 063504 [hep-ph/9809453] [INSPIRE].

[86] G.F. Giudice, E.W. Kolb and A. Riotto, Largest temperature of the radiation era and its cosmological implications, Phys. Rev. D 64 (2001) 023508 [hep-ph/0005123] [InSPIRE].

[87] R.T. Co, F. D'Eramo, L.J. Hall and D. Pappadopulo, Freeze-in dark matter with displaced signatures at colliders, JCAP 12 (2015) 024 [arXiv: 1506.07532] [INSPIRE].

[88] J.L. Feng, S. Iwamoto, Y. Shadmi and S. Tarem, Long-lived sleptons at the LHC and a 100 TeV proton collider, JHEP 12 (2015) 166 [arXiv: 1505.02996] [INSPIRE].

[89] CMS collaboration, Search for long-lived charged particles in proton-proton collisions at $\sqrt{s}=13$ TeV, CMS-PAS-EXO-15-010 (2015). 
[90] CMS collaboration, Measurement of the top quark pair production cross section in proton-proton collisions at $\sqrt{s}=13$ TeV, Phys. Rev. Lett. 116 (2016) 052002 [arXiv: 1510.05302] [INSPIRE].

[91] J.M. Campbell, J.W. Huston and W.J. Stirling, Hard interactions of quarks and gluons: a primer for LHC physics, Rept. Prog. Phys. 70 (2007) 89 [hep-ph/0611148] [InSPIRE].

[92] Y. Bai and J. Berger, Lepton portal dark matter, JHEP 08 (2014) 153 [arXiv:1402.6696] [INSPIRE].

[93] N.D. Christensen and C. Duhr, FeynRules - Feynman rules made easy, Comput. Phys. Commun. 180 (2009) 1614 [arXiv:0806.4194] [INSPIRE].

[94] P.W. Graham, D.E. Kaplan, S. Rajendran and P. Saraswat, Displaced supersymmetry, JHEP 07 (2012) 149 [arXiv: 1204.6038] [INSPIRE].

[95] L. Kofman, A.D. Linde and A.A. Starobinsky, Towards the theory of reheating after inflation, Phys. Rev. D 56 (1997) 3258 [hep-ph/9704452] [INSPIRE].

[96] A. Monteux and C.S. Shin, Thermal Goldstino production with low reheating temperatures, Phys. Rev. D 92 (2015) 035002 [arXiv: 1505.03149] [INSPIRE]. 